\title{
On arithmetic progressions in non-periodic self-affine tilings
}

\author{
Yasushi Nagai * $\quad$ Shigeki Akiyama $^{\dagger} \quad$ Jeong-Yup Lee ${ }^{\ddagger}$
}

March 31, 2021

\begin{abstract}
We study the repetition of patches in self-affine tilings in $\mathbb{R}^{d}$. In particular, we study the existence and non-existence of arithmetic progressions. We first show that an arithmetic condition of the expansion map for a self-affine tiling implies the non-existence of certain one-dimensional arithmetic progressions. Next, we show that the existence of full-rank infinite arithmetic progressions, pure discrete dynamical spectrum, and limit periodicity are all equivalent for a certain class of self-affine tilings. We finish by giving a complete picture for the existence/non-existence of full-rank infinite arithmetic progressions in the self-similar tilings in $\mathbb{R}^{d}$.
\end{abstract}

\section{Introduction}

A tiling is a cover of the Euclidean space $\mathbb{R}^{d}$ by a set of tiles without interior overlaps. The simplest class of tilings is the one of crystallographic tilings, where a tiling $\mathcal{T}$ is crystallographic if its symmetry group is a crystallographic group, i.e. it has translational symmetry $\mathcal{T}+x=\mathcal{T}$ for the vectors $x$ in a basis of $\mathbb{R}^{d}$. Although the crystallographic tilings are interesting, the discovery of quasicrystals requires us to go beyond that category and study non-periodic tilings, that is, tilings without translational symmetry. As models of quasicrystals, the non-periodic tilings that are "ordered" are important, although there are several interpretations of the term "ordered". The most important interpretation is that the tiling is pure point diffractive, which by definition means that the diffraction measure is pure point. (The diffraction measure models physical diffraction pattern. The presence of point masses is an indication of "order" for the tiling. For an introduction of diffraction spectrum, see for example [3, chapter 9].) Being pure point diffractive is

\footnotetext{
${ }^{*}$ School of Mathematics and Statistics, Faculty of Science, Technology, Engineering and Mathematics, The Open University, Walton Hall, Milton Keynes, MK7 6AA, UK, e-mail: yasushi.nagai@open.ac.uk

${ }^{\dagger}$ Institute of Mathematics, University of Tsukuba, 1-1-1 Tennodai, Tsukuba, Ibaraki 305-8571, Japan, e-mail:akiyama@math.tsukuba.ac.jp

${ }^{\ddagger}$ Department of Mathematics Education, Catholic Kwandong University, Gangneung, Gangwon 210-701, Korea, or KIAS, 85 Hoegiro, Dongdaemun-gu, Seoul 02455, Korea, e-mail:jylee@cku.ac.kr
} 
equivalent to having pure discrete dynamical spectrum [6]. (We will define pure discrete dynamical spectrum in page 6.) Being pure point diffractive is also equivalent to being almost periodic in the sense of Gouéré [11] and to being mean almost periodic [17]. These forms of almost periodicity characterize how patterns (patches) distribute in the tiling. This is why the distribution of patches in a given tiling, especially the repetition of finite patches, is important.

Another interpretation of the term "ordered" is that the tiling is limit-periodic. Here, a tiling is said to be limit-periodic if, except for a set of zero-density tiles, all tiles $T$ repeat crystallographically, which means that there is a lattice $L$ of $\mathbb{R}^{d}$ for each $T$ such that the all translates $T+x, x \in L$, are included in the tiling. We usually further assume that the lattice $L$ which appears in this way belongs to a decreasing sequence of lattices. This is a geometric version of Toeplitz sequences [13. The period doubling tiling and the chair tilings are well-known examples of limit-periodic tiling.

In this paper, we deal with a type of the repetition of patches, that is, arithmetic progressions of patches, and discuss its relations with pure point diffraction and limitperiodicity for self-affine tilings. A self-affine tiling is a tiling with an inflation-subdivision symmetry, which means that there exists an expansive linear map $Q$ that makes all tiles larger and we can subdivide them into the tiles of original size to obtain the original tiling. Illustrative examples are found in Figure 1, where we show a chair tiling and a Robinson triangle tiling, respectively. The latter is essentially the same (MLD) as one of the famous Penrose tilings [3, Section 6.2].

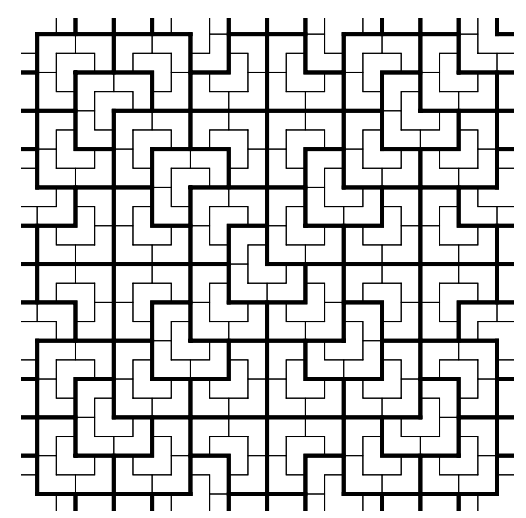

(a) a chair tiling

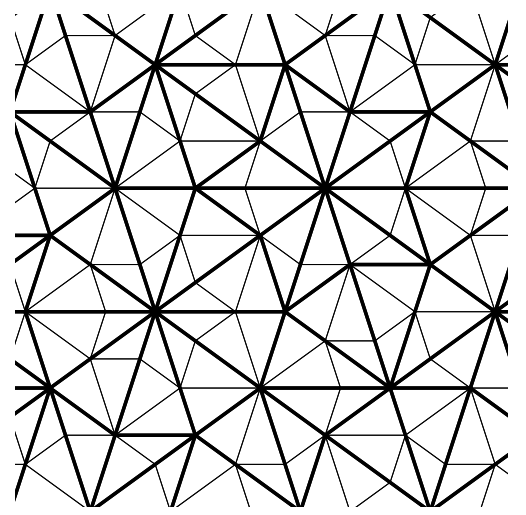

(b) a Robinson triangle tiling

Figure 1: Self-affine tiling

A one-dimensional arithmetic progression of a patch $\mathcal{P}$ is a patch of the form $\bigcup_{k=1}^{n} \mathcal{P}+$ $k x$, where $x$ is a non-zero vector of $\mathbb{R}^{d}$. Here, $n$ is either a positive integer or $\infty$. This is a pattern where the patch $\mathcal{P}$ appears $n$ times, separated in each instance by the translation vector $x$ from the previous. It is interesting to observe that a chair tiling possess such 
an arithmetic progression in the diagonal direction, as we see in Figure 1. In this article, we first give an arithmetic condition on the expansion map $Q$ for self-affine tilings which implies the non-existence of arbitrarily long arithmetic progressions for fixed $\mathcal{P}$ and $x$ (Theorem 3.1, Theorem 3.6). For example, in Penrose tilings, the arithmetic progressions stop at a certain finite $n$ which depends on each $\mathcal{P}$ and $x$. This is done in Section 3 ,

Next, in Section 4, we deal with full-rank infinite arithmetic progressions, which are by definition patches of the form

$$
\bigcup_{\left(l_{1}, l_{2}, \ldots l_{d}\right) \in \mathbb{Z}^{d}} \mathcal{P}+\sum_{j=1}^{d} l_{j} b_{j},
$$

where $\left\{b_{1}, b_{2}, \ldots b_{d}\right\}$ is a basis of $\mathbb{R}^{d}$. Interestingly, for a class of self-affine tilings, the existence of full-rank arithmetic progressions is equivalent to having pure discrete dynamical spectrum and also to being limit periodic (Theorem 4.7, Corollary 4.19).

In Section 5, we give a complete picture for the existence/non-existence of full-rank infinite arithmetic progressions in a self-similar tiling in $\mathbb{R}^{d}$. We then give further problems in Section 6 and finish the article with Appendix, where some of the proofs are given.

All of this is done after introducing the necessary notation and recalling known results in Section 2 ,

\section{Notation and known results}

In this section, we recall relevant notation and known results. In the whole article, $B(x, R)$ is the closed ball in the $d$-dimensional Euclidean space $\mathbb{R}^{d}$ with its center $x \in \mathbb{R}^{d}$ and radius $R>0$. Often $B(0, R)$ is denoted by $B_{R}$. The symbol $\mathbb{T}$ denotes the one-dimensional torus: $\mathbb{T}=\{z \in \mathbb{C}|| z \mid=1\}$.

\section{$2.1 \quad$ Tilings and substitutions}

Let $L$ be a finite set. An $L$-labeled tile is a pair $(S, l)$ of a compact non-empty subset $S$ of $\mathbb{R}^{d}$, such that $\overline{S^{\circ}}=S$ (the closure of the interior coincides with the original $S$ ), and an $l \in L$. We often fix $L$ and call $L$-labeled tiles just tiles. For a tile $T=(S, l)$, we write $S=\operatorname{supp} T$ and $l=l(T)$. We also write $\operatorname{int}(T)=(\operatorname{supp} T)^{\circ}$. For a $T=(S, l)$ and an $x \in \mathbb{R}^{d}$, we set $T+x=(S+x, l)$.

Alternatively, we also call a compact non-empty subset $S$ of $\mathbb{R}^{d}$ such that $S=\overline{S^{\circ}}$ a tile. Thus "a tile" means either a "labeled" or an "un-labeled" tile. Both types of tiles are useful in aperiodic order. For example, if we consider the geometric realization of a constant-length symbolic substitution, we have to give labels to tiles in order to distinguish the intervals for different letters. On the other hand, for many geometric substitutions 
labels are not necessary. 11 For an unlabeled tile $S$, we also use the notation $\operatorname{supp} S=S$ and $\operatorname{int}(S)=S^{\circ}$.

A set $\mathcal{P}$ of tiles is called a patch if for any $T_{1}, T_{2} \in \mathcal{P}$ with $\operatorname{int}\left(T_{1}\right) \cap \operatorname{int}\left(T_{2}\right) \neq \emptyset$, we have $T_{1}=T_{2}$. For a patch $\mathcal{P}$, its support $\operatorname{supp} \mathcal{P}$ is defined by $\operatorname{supp} \mathcal{P}=\overline{\bigcup_{T \in \mathcal{P}} \operatorname{supp} T}$. If a patch $\mathcal{T}$ satisfies the condition $\operatorname{supp} \mathcal{T}=\mathbb{R}^{d}$, we call $\mathcal{T}$ a tiling. Often patches are assumed to be finite sets, but in this article we do not assume that. (Infinite patches that are not tilings appear in this article.) A patch that is a finite set is called a finite patch. Given a tiling $\mathcal{T}$, a patch $\mathcal{P}$ is said to be $\mathcal{T}$-legal if there is an $x \in \mathbb{R}^{d}$ such that $\mathcal{P}+x \subset \mathcal{T}$, where for a patch $\mathcal{P}$ in $\mathbb{R}^{d}$ and an $x \in \mathbb{R}^{d}$, we set $\mathcal{P}+x=\{T+x \mid T \in \mathcal{P}\}$. A vector $x \in \mathbb{R}^{d}$ such that there is a $T \in \mathcal{T}$ with $T+x \in \mathcal{T}$ is called a return vector for $\mathcal{T}$. The set of all return vectors for $\mathcal{T}$ is denoted by $\Xi(\mathcal{T})$.

For a patch $\mathcal{P}$ and a subset $S$ of $\mathbb{R}^{d}$, we define a new patch $\mathcal{P} \sqcap S$ via

$$
\mathcal{P} \sqcap S=\{T \in \mathcal{P} \mid \operatorname{supp} T \cap S \neq \emptyset\} .
$$

Define another patch $\mathcal{P} \wedge S$ via

$$
\mathcal{P} \wedge S=\{T \in \mathcal{P} \mid \operatorname{supp} T \subset S\}
$$

Next, we define the densities for patches. We first define van Hove sequences.

Definition 2.1. A sequence $\left(A_{n}\right)_{n=1,2, \cdots}$ of measurable subsets of $\mathbb{R}^{d}$ with positive Lebesgue measures is called a van Hove sequence if, for each compact $K \subset \mathbb{R}^{d}$, we have

$$
\lim _{n} \frac{\operatorname{vol}\left(\partial^{K} A_{n}\right)}{\operatorname{vol}\left(A_{n}\right)}=0
$$

where vol denotes the Lebesgue measure and

$$
\partial^{K} A_{n}=\left(\left(K+A_{n}\right) \backslash A_{n}^{\circ}\right) \cup\left(\left(-K+\overline{\mathbb{R}^{d} \backslash A_{n}}\right) \cap A_{n}\right) .
$$

The density dens $\left(A_{n}\right) \mathcal{P}$ of a patch $\mathcal{P}$ with respect to a van Hove sequence $\left(A_{n}\right)_{n}$ is defined via

$$
\operatorname{dens}_{\left(A_{n}\right)_{n}} \mathcal{P}=\limsup _{n \rightarrow \infty} \frac{1}{\operatorname{vol}\left(A_{n}\right)} \operatorname{vol}\left((\operatorname{supp} \mathcal{P}) \cap A_{n}\right)
$$

We say that a patch $\mathcal{P}$ has zero density if, for any van Hove sequence $\left(A_{n}\right)_{n}$, the density $\operatorname{dens}_{\left(A_{n}\right)_{n}} \mathcal{P}$ is zero.

\footnotetext{
${ }^{1}$ Sometimes it is not a good idea to use $\left\{\left(A_{i}, i\right) \mid i=1,2, \ldots n\right\}$ as the alphabet of a geometric substitution, since this does not describe the rotational symmetry of a substitution $(\omega(R(P))=R(\omega(P))$, where $P$ is a proto-tile and $R$ is a rotation), which we often use tacitly. It is sometimes more convenient to use unlabeled tiles and sometimes labeled ones, and so it is useful to include both types of tiles in the definition of tiles.
} 
If the diameters of the tiles in $\mathcal{P}$ are bounded from above by some uniform constant, we have

$$
\begin{aligned}
\operatorname{dens}_{\left(A_{n}\right)_{n}} \mathcal{P} & =\limsup _{n \rightarrow \infty} \frac{1}{\operatorname{vol}\left(A_{n}\right)} \operatorname{vol}\left(\operatorname{supp}\left(\mathcal{P} \sqcap A_{n}\right)\right) \\
& =\limsup _{n \rightarrow \infty} \frac{1}{\operatorname{vol}\left(A_{n}\right)} \operatorname{vol}\left(\operatorname{supp}\left(\mathcal{P} \wedge A_{n}\right)\right) .
\end{aligned}
$$

The corresponding dynamical system for a tiling is an important object. To define it, we need to define the local matching topology on the set of all patches, as follows: for patches $\mathcal{P}_{1}$ and $\mathcal{P}_{2}$, we define a set $\Delta\left(\mathcal{P}_{1}, \mathcal{P}_{2}\right)$ to be the set of all real numbers $\varepsilon$ between 0 and $\frac{1}{\sqrt{2}}$ such that there are $x_{1}, x_{2} \in B_{\varepsilon}$ with

$$
\left(\mathcal{P}_{1}+x_{1}\right) \sqcap B_{1 / \varepsilon}=\left(\mathcal{P}_{2}+x_{2}\right) \sqcap B_{1 / \varepsilon} .
$$

Define a metric $\rho$ on the space of all patches in $\mathbb{R}^{d}$ via

$$
\rho\left(\mathcal{P}_{1}, \mathcal{P}_{2}\right)=\inf \Delta\left(\mathcal{P}_{1}, \mathcal{P}_{2}\right) \cup\left\{\frac{1}{\sqrt{2}}\right\}
$$

Here, the choice of the number $1 / \sqrt{2}$ makes it easier to prove the triangle inequality. The topology defined by $\rho$ is called the local matching topology. If we instead use $\wedge$ as a cutting-off operation, the resulting topology is the same on a large space of patches. It is known that this metric is complete $([22])$.

Given a tiling $\mathcal{T}$, define the continuous hull $X_{\mathcal{T}}$ via

$$
X_{\mathcal{T}}=\overline{\left\{\mathcal{T}+x \mid x \in \mathbb{R}^{d}\right\}}
$$

where the closure is taken with respect to the local matching topology. There is a necessary and sufficient condition, finite local complexity ( $F L C)$, for the continuous hull to be compact. A tiling $\mathcal{T}$ has FLC if, for each compact $K \subset \mathbb{R}^{d}$, the set

$$
\left\{\mathcal{T} \sqcap(K+x) \mid x \in \mathbb{R}^{d}\right\}
$$

is finite up to translation. (That is, if we identify two patches that are translates of each other, then the set is finite). The FLC of a tiling is equivalent to the compactness of its continuous hull ([3, Proposition 5.4]). By taking $K=\{0\}$, we also see the FLC implies that there are only finitely many tiles in $\mathcal{T}$ up to translation. The group $\mathbb{R}^{d}$ acts continuously on $X_{\mathcal{T}}$ via

$$
X_{\mathcal{T}} \times \mathbb{R}^{d} \ni(\mathcal{S}, x) \mapsto \mathcal{S}+x \in X_{\mathcal{T}}
$$

The pair of $X_{\mathcal{T}}$ and this action is called the tiling dynamical system associated with $\mathcal{T}$. The tiling $\mathcal{T}$ is said to be repetitive if, for any finite $\mathcal{T}$-legal patch $\mathcal{P}$, the translates of $\mathcal{P}$ 
appear in $\mathcal{T}$ with a uniformly bounded gap. If $\mathcal{T}$ is repetitive, then the tiling dynamical system $\left(X_{\mathcal{T}}, \mathbb{R}^{d}\right)$ is minimal ([3, Proposition 5.4]), that is, every orbit is dense.

In this article, we assume that all tilings which appear have FLC, and there is one and only one invariant probability measure $\mu$ for each tiling dynamical system. The self-affine tilings defined via a primitive FLC substitutions, which we focus on in this article, satisfy this assumption.

If, for a vector $a \in \mathbb{R}^{d}$, there is a non-zero vector $f \in L^{2}(\mu)$ such that for each $x \in \mathbb{R}^{d}$, the two maps

$$
\begin{array}{r}
X_{\mathcal{T}} \ni \mathcal{S} \mapsto f(\mathcal{S}-x), \\
X_{\mathcal{T}} \ni \mathcal{S} \mapsto e^{2 \pi i\langle x, a\rangle} f(\mathcal{S})
\end{array}
$$

coincide $\mu$-almost everywhere, then we call $a$ an eigenvalue for the tiling dynamical system $\left(X_{\mathcal{T}}, \mathbb{R}^{d}\right)$. Here, $\langle\cdot, \cdot\rangle$ is the standard Euclidean inner product. In this case the function $f$ is called an eigenfunction. If the function $f$ can be chosen to be continuous, we call $a$ a topological eigenvalue and $f$ a continuous eigenfunction. We say that $\mathcal{T}$ has pure discrete dynamical spectrum if there is a complete orthonormal basis for the Hilbert space $L^{2}(\mu)$ consisting of eigenfunctions.

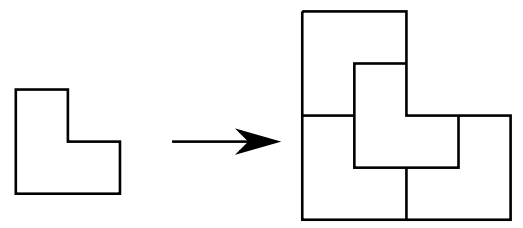

Figure 2: the chair substitution

One can construct interesting tilings via substitution rules. Intuitively, a substitution rule is a recipe for "expanding a tile, followed by subdividing it so that we obtain a patch." (See Figure 2. This is an example of substitution rule, called the chair substitution, that generates chair tilings in Figure 1,) A substitution rule by definition consists of

- an alphabet $\mathcal{A}$, which is a finite set consisting of tiles in $\mathbb{R}^{d}$,

- an expansion map $Q$, which is a linear transformation $Q: \mathbb{R}^{d} \rightarrow \mathbb{R}^{d}$ whose eigenvalues are all greater than 1 in absolute values, and

- a map $\omega$ which sends each element $P$ of $\mathcal{A}$ to a patch $\omega(P)$ consisting of translates of elements of $\mathcal{A}$ such that

$$
\operatorname{supp} \omega(P)=Q(\operatorname{supp} P)
$$


Often the map $\omega$ is also called a substitution rule. The elements of $\mathcal{A}$ are called proto-tiles. For the example of the chair substitution, the alphabet $\mathcal{A}$ consists of the tile on the lefthand side of Figure 2 and its rotations by degrees 90, 180, 270. $Q=2 I$ (where $I$ is the identity matrix) and the map $\omega$ sends each element of $\mathcal{A}$ to the patch that is obtained in the way depicted in Figure 2.

Given a substitution rule $\omega$ with an indexed alphabet $\mathcal{A}=\left\{T_{1}, T_{2}, \ldots, T_{n}\right\}$, we associate a substitution matrix $M_{\omega}$ whose $(i, j)$-element is the number of the occurrences of the tile $T_{i}$ in $\omega\left(T_{j}\right)$. The substitution $\omega$ is said to be primitive if the matrix $M_{\omega}$ is primitive, which means that its sufficiently large powers have only non-zero elements. The substitution $\omega$ is said to be irreducible if the characteristic polynomial of $M_{\omega}$ is irreducible.

Given an $\omega$, we can define, in a natural way, a patch $\omega(\mathcal{P})$ for a patch $\mathcal{P}$ consisting of translates of tiles in $\mathcal{A}$, by applying the same "expanding and subdividing" rule to each tile in $\mathcal{P}$. We can iterate $\omega$ and sometimes, as $n \rightarrow \infty, \omega^{n}(\mathcal{P})$ grows larger and larger and converges to a tiling. Intuitively, a tiling constructed in this way is called a self-affine tiling, of which formal definition is given below. If a tiling $\mathcal{T}$ consists of translates of tiles in $\mathcal{A}$ and we have $\omega(\mathcal{T})=\mathcal{T}$, we call $\mathcal{T}$ a fixed point of the substitution rule $\omega$. If moreover $\mathcal{T}$ has FLC and is repetitive, we call $\mathcal{T}$ a self-affine tiling. If the expansion map is of the form $\lambda I$ for some $\lambda>1$, where $I$ is the identity map, then the resulting self-affine tiling is called a self-similar tiling. In this case, the number $\lambda$ is called the expansion factor. Some chair tilings are self-similar tilings with expansion factor 2.

For a substitution rule $\omega$ of $\mathbb{R}^{d}$, we say $\omega$ has FLC if for each compact $K \subset \mathbb{R}^{d}$, the set

$$
\left\{\omega^{n}(P) \sqcap(K+x) \mid n>0, P \in \mathcal{A}, x \in \mathbb{R}^{d}\right\}
$$

is finite up to translation. If $\omega$ has FLC, any repetitive fixed points for $\omega$ have FLC.

In general, given a tiling $\mathcal{T}$ of $\mathbb{R}^{d}$ with FLC, we can obtain a discrete and closed subset $D$ of $\mathbb{R}^{d}$ by choosing one representative point from the support of each tile in $\mathcal{T}$, in such a way that the relative positions of representative points for translationally equivalent tiles are always the same. This $D$ is an example of Delone set, because (1)distances between any two points are uniformly bounded from below, and (2)there is an $R>0$ such that any balls of $\mathbb{R}^{d}$ with radius $R$ contain points of $D$. If $\mathcal{T}$ is a self-affine tiling associated with a substitution rule $\omega$ with an expansion map $Q$, we can take a control point from each tile as a representative point [24]: we first choose a tile map $f: \mathcal{T} \rightarrow \mathcal{T}$ such that $f(T) \in \omega(T)$ for each $T \in \mathcal{T}$, and let $c(T) \in \operatorname{supp} T$ be the unique point in $\bigcap_{n=1}^{\infty} Q^{-n}\left(f^{n}(T)\right)$. The resulting Delone set $D=\{c(T) \mid T \in \mathcal{T}\}$ is well-behaved in the sense that (1) we have $Q(D) \subset D$ (because $Q(c(T))=c(f(T))$ ), and (2) with an additional condition on $\mathcal{T}$, the set $D$ is a Meyer set, which means that for some neighborhood $U$ of $0 \in \mathbb{R}^{d}$, we have

$$
((D-D)-(D-D)) \cap U=\{0\} .
$$

The tilings $\mathcal{T}$ with the latter property (2) for some choice of representative points are said to have the Meyer property. 
A condition for a self-affine tiling $\mathcal{T}$ to have the Meyer property is given in [16]. We say that a set $\Lambda$ of algebraic integers forms a Pisot family if for any $\lambda \in \Lambda$ and any algebraic conjugate $\mu$ of $\lambda$ with $|\mu| \geq 1$, we have $\mu \in \Lambda$.

Theorem 2.2 ([16]). Let $\mathcal{T}$ be a self-affine tiling in $\mathbb{R}^{d}$ with an expansion map $Q$. Suppose $Q$ is diagonalizable over $\mathbb{C}$ and all the eigenvalues are algebraic conjugates and have the same multiplicity. Then $\mathcal{T}$ has the Meyer property if and only if $\operatorname{sp}(Q)$ is a Pisot family, where $\operatorname{sp}(Q)$ is the set of all eigenvalues for $Q$.

\subsection{Arithmetic progressions in tilings}

In this subsection we define arithmetic progressions in tilings and describe known results for them.

Definition 2.3. Let $\mathcal{T}$ be a tiling in $\mathbb{R}^{d}, \mathcal{P}$ a non-empty finite patch, $n$ an integer greater than 0 and $x$ a vector in $\mathbb{R}^{d} \backslash\{0\}$. A set of tiles of the form

$$
\bigcup_{k=1}^{n} \mathcal{P}+k x
$$

is called a (one-dimensional) arithmetic progression of length $n$. A set of tiles of the form

$$
\bigcup_{k=1}^{\infty} \mathcal{P}+k x
$$

or

$$
\bigcup_{k=-\infty}^{\infty} \mathcal{P}+k x
$$

is called a (one-dimensional) infinite arithmetic progression.

Let $\mathcal{B}=\left\{b_{1}, b_{2}, \ldots, b_{d}\right\}$ be a basis of $\mathbb{R}^{d}$, which is regarded as a vector space over $\mathbb{R}$. A set of tiles of the form

$$
\bigcup_{\left(l_{1}, l_{2}, \cdots, l_{d}\right) \in \mathbb{Z}^{d}} \mathcal{P}+\sum_{i=1}^{d} l_{i} b_{i}
$$

is called a full-rank infinite arithmetic progression.

In this paper, we discuss the existence/non-existence of various arithmetic progressions in various tilings. We first note that the following two lemmas hold:

Lemma 2.4. Let $\mathcal{T}$ be a tiling in $\mathbb{R}^{d}$ that has FLC. Let $\mathcal{P}$ be a non-empty, finite and $\mathcal{T}$-legal patch and take an $x \in \mathbb{R}^{d} \backslash\{0\}$. Then the following conditions are equivalent: 
1. for each $n>0$, an arithmetic progression $\bigcup_{k=1}^{n} \mathcal{P}+k x$ is $\mathcal{T}$-legal.

2. for some $\mathcal{S} \in X_{\mathcal{T}}$, an infinite arithmetic progression $\bigcup_{k=1}^{\infty} \mathcal{P}+k x$ is $\mathcal{S}$-legal.

3. for some $\mathcal{S} \in X_{\mathcal{T}}$, an infinite arithmetic progression $\bigcup_{k=-\infty}^{\infty} \mathcal{P}+k x$ is $\mathcal{S}$-legal.

Proof. If the first condition is satisfied, we can take a sequence $x_{1}, x_{2}, \ldots \in \mathbb{R}^{d}$ such that for each $n$, the translate $\mathcal{T}-x_{n}$ includes $\bigcup_{k=1}^{n} \mathcal{P}+k x$. By FLC, the hull $X_{\mathcal{T}}$ is compact and we can take a subsequence $\left(x_{n_{j}}\right)_{j}$ such that $\mathcal{S}=\lim _{j \rightarrow \infty} \mathcal{T}-x_{n_{j}}$ is convergent. This $\mathcal{S}$ includes the patch $\bigcup_{k=1}^{\infty} \mathcal{P}+k x$. We can also take an $x_{n}$ such that $\mathcal{T}-x_{n}$ includes $\bigcup_{k=-n}^{n} \mathcal{P}+k x$. By the same argument, we can find an $\mathcal{S} \in X_{\mathcal{T}}$ that includes $\bigcup_{k=-\infty}^{\infty} \mathcal{P}+k x$. We have proved that the first condition implies both the second and the third condition.

Conversely, if the second or the third condition is satisfied, then for each $n$, the patch $\bigcup_{k=1}^{n} \mathcal{P}+k x$ is $\mathcal{S}$-legal. Since $\mathcal{S}$ can be approximated by translates of $\mathcal{T}$, we see $\bigcup_{k=1}^{n} \mathcal{P}+k x$ is $\mathcal{T}$-legal for each $n$.

Lemma 2.5. Let $\mathcal{T}$ be a tiling in $\mathbb{R}^{d}$ that has $F L C$ and is repetitive. Let $\mathcal{P}$ be a nonempty, finite and $\mathcal{T}$-legal patch and $\mathcal{B}=\left\{b_{1}, b_{2}, \ldots b_{d}\right\}$ be a basis of $\mathbb{R}^{d}$. Then the following conditions are equivalent:

1. for each $n>0$, the set

$$
\bigcup_{\left(l_{1}, l_{2}, \ldots, l_{d}\right) \in\{1,2, \ldots, n\}^{d}} \mathcal{P}+\sum_{i=1}^{d} l_{i} b_{i}
$$

is a $\mathcal{T}$-legal patch.

2. The infinite arithmetic progression

$$
\bigcup_{\left(l_{1}, l_{2}, \ldots, l_{d}\right) \in \mathbb{Z}^{d}} \mathcal{P}+\sum_{i=1}^{d} l_{i} b_{i}
$$

is $\mathcal{S}$-legal for any $\mathcal{S} \in X_{\mathcal{T}}$.

Proof. If the first condition is satisfied, there is an $x_{n} \in \mathbb{R}^{d}$ for $n=1,2, \cdots$ such that $\mathcal{T}-x_{n}$ contains a patch

$$
\bigcup_{\left(l_{1}, l_{2}, \ldots, l_{d}\right) \in\{-n,-n+1, \ldots, n\}^{d}} \mathcal{P}+\sum_{i=1}^{d} l_{i} b_{i} .
$$

Since $X_{\mathcal{T}}$ is a compact metric space, we can take $n_{1}<n_{2}<n_{3}<\cdots$ such that $\mathcal{T}-x_{n_{j}}$ converges as $j \rightarrow \infty$ to some $\mathcal{S} \in X_{\mathcal{T}}$. This $\mathcal{S}$ contains (5). Since the tiling dynamical system $\left(X_{\mathcal{T}}, \mathbb{R}^{d}\right)$ is minimal, for each $\mathcal{R} \in X_{\mathcal{T}}$ there is a sequence $y_{1}, y_{2}, \cdots \in \mathbb{R}^{d}$ such that $\lim _{m \rightarrow \infty} \mathcal{S}+y_{m}=\mathcal{R}$. We can take a subsequence $\left(y_{m_{j}}\right)_{j}$ that is convergent $\bmod \operatorname{span}_{\mathbb{Z}} \mathcal{B}$. We still have $\mathcal{R}=\lim _{j \rightarrow \infty} \mathcal{S}+y_{m_{j}}$, and this $\mathcal{R}$ contains a translate of the infinite arithmetic progression (5). 
Remark 2.6. Notice that (1)for a repetitive $\mathcal{T}$, the existence/non-existence of a onedimensional finite arithmetic progression $\bigcup_{k=1}^{n} \mathcal{P}+k x$ does not depend on the choice of $\mathcal{S} \in X_{\mathcal{T}}$, because $X_{\mathcal{T}}$ coincides with the local isomorphism class [3, Proposition 5.4], (2)the existence/non-existence of a one-dimensional infinite arithmetic progression $\bigcup_{k=1}^{\infty} \mathcal{P}+k x$ may depend on the choice of the tiling from the hull (compare Lemma 2.4), and (3)the existence/non-existence of the full-rank infinite arithmetic progressions does not (Lemma 2.5). In this paper, we discuss the existence/non-existence of finite arithmetic progressions (1) and of full-rank infinite arithmetic progressions (3). We discuss the existence and nonexistence inside a repetitive fixed point $\mathcal{T}$ of a substitution which we initially consider, but the same existence/non-existence applies for any $\mathcal{S} \in X_{\mathcal{T}}$.

Example 2.7. Let $\omega$ be the chair substitution. For $\mathcal{P}_{0}$ and $x \neq 0$, as depicted in Figure 3, there is an arithmetic progression of length $2^{k}$ at the $k$-th step of the substitution. This means that the chair tilings satisfy the equivalent conditions of Lemma 2.4 for this $\mathcal{P}_{0}$ and $x$. If we pick an arbitrary finite patch $\mathcal{P}$ that appears in chair tilings, since a translate of $\mathcal{P}$ is included in $\omega^{l}\left(\mathcal{P}_{0}\right)$ for some $l>0$, we see for $\mathcal{P}$ and $2^{l} x$, there are arbitrarily long (one-dimensional) arithmetic progressions in chair tilings.

We can see that the chair tilings admit full-rank infinite arithmetic progressions by considering the modification of the chair tilings into tilings with decorated squares $[5$, Section 4]. The supertiles of the decorated squares have common tiles except for the main diagonals. Since the supertiles are aligned in a translate of $2^{n} \mathbb{Z}^{2}$, if we chose tiles from supertiles that are relatively the same position and are outside the main diagonals, we can find a full-rank infinite arithmetic progressions.
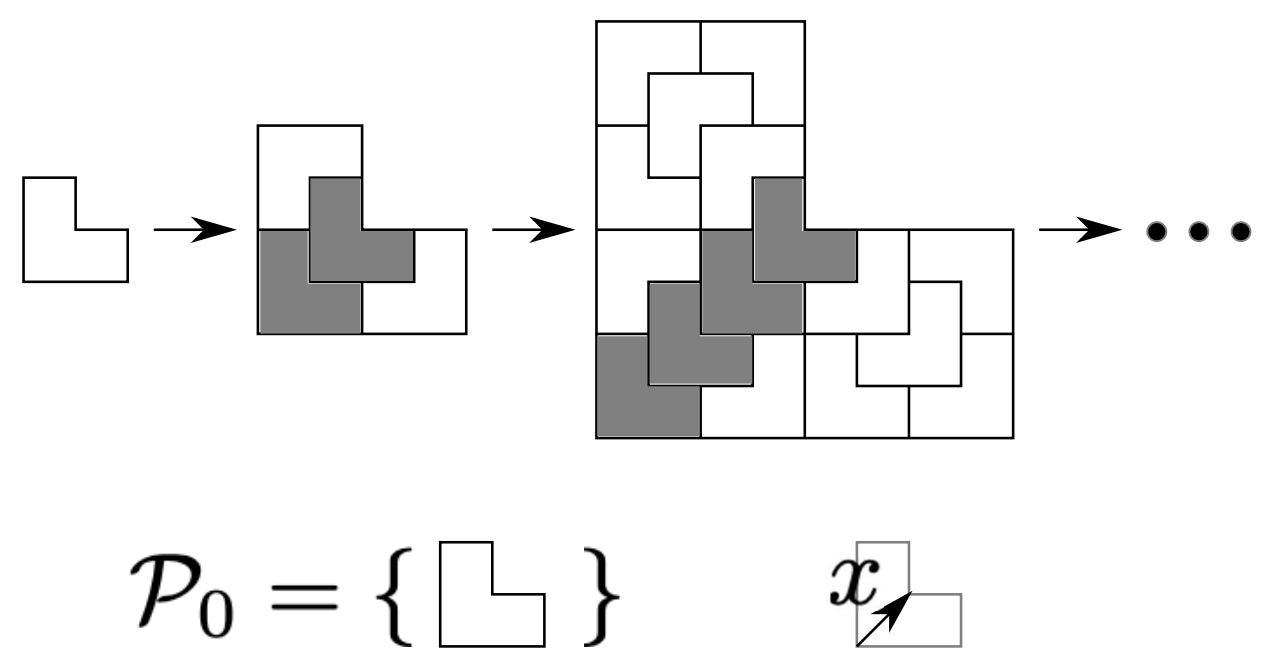

Figure 3: Arbitrarily long arithmetic progressions in chair tilings 
Example 2.8. Let $\omega$ be the table substitution ([3, Example 6.2]). As is seen at the center of Figure 4, there are arbitrarily long finite arithmetic progressions (in the horizontal direction) in the self-similar tilings for $\omega$. However, we will see by using Theorem 4.7 that there are no full-rank infinite arithmetic progressions in those tilings, since they do not have pure discrete dynamical spectrum ([27, Example 7.3]).

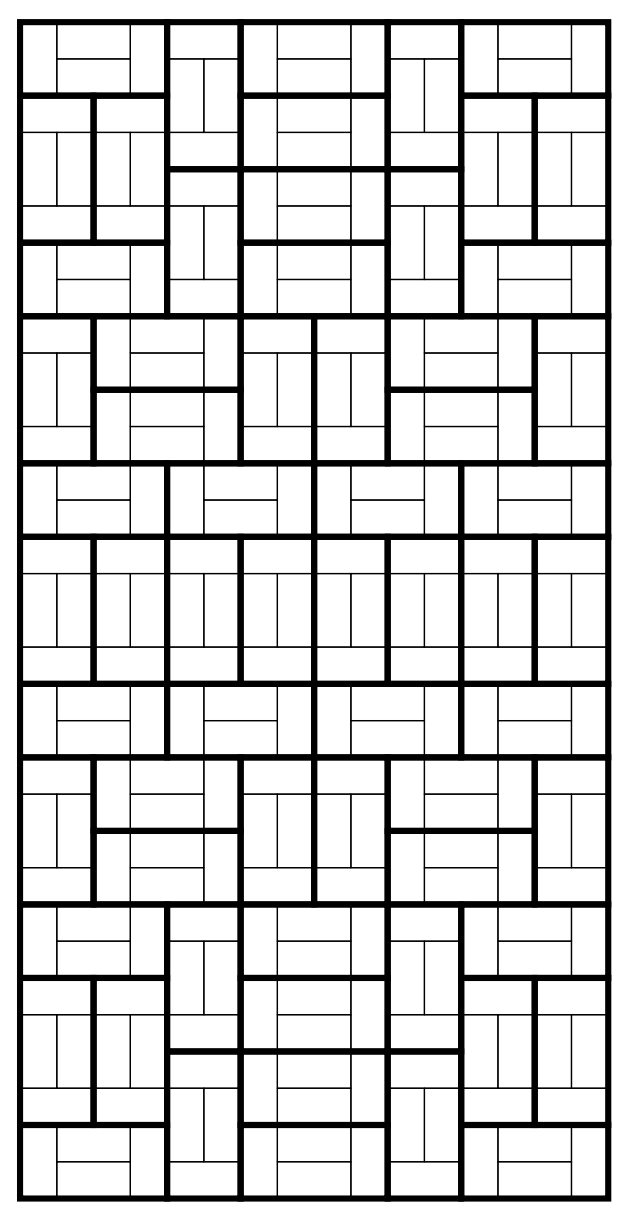

Figure 4: A patch generated by the table substitution

To prove the existence of arithmetic progressions in tilings, the following theorem is useful:

Theorem 2.9 ([8], Theorem 2.6). Let $X$ be a compact metric space and $T_{1}, T_{2}, \ldots, T_{l}$ commuting continuous maps of $X$ to itself. Then there is a point $x \in X$ and a sequence $n_{k} \rightarrow \infty$ such that $T_{i}^{n_{k}} x \rightarrow x$ simultaneously for $i=1,2, \ldots, l$. 
Using this multiple Birkhoff recurrence theorem, the authors of [20] proved the following theorem:

Theorem 2.10 ([20], Theorem 2). Let $\mathcal{T}$ be a tiling in $\mathbb{R}^{d}$ that has FLC. Given an $\varepsilon>0$ and a finite set $F \subset \mathbb{R}^{d}$, there exist an $m \in \mathbb{Z}_{>0}$ and a patch $\mathcal{P}$ such that

1. the support of $\mathcal{P}$ covers a ball of radius $1 / \varepsilon$, and

2. for each $u \in F$ there is a vector $c \in \mathbb{R}^{d}$ such that $\|c\|<\varepsilon$ and

$$
\mathcal{P}+m u+c \subset \mathcal{T}
$$

Given a tiling $\mathcal{T}$ in $\mathbb{R}^{d}$ of FLC, an $x \in \mathbb{R}^{d} \backslash\{0\}$ and an $n>0$, by applying Theorem 2.10 for $F=\{0, x, 2 x, \ldots,(n-1) x\}$ and an $\varepsilon>0$, we see that there is a patch $\mathcal{P}$ that covers a ball of radius $1 / \varepsilon$ and an $m \in \mathbb{Z}_{>0}$ such that an "almost arithmetic progression"

$$
\bigcup_{k=1}^{n} \mathcal{P}+k m x+c_{k}
$$

is $\mathcal{T}$-legal. ("Almost" means that there is an error term $c_{k}$ with $\left\|c_{k}\right\|<\varepsilon$.)

If we start with a non-empty finite patch $\mathcal{Q}$ and assume that $\mathcal{T}$ is repetitive, then if $\varepsilon$ is small enough every patch of $\mathcal{T}$ that covers a ball of radius $1 / \varepsilon$ contains a translate of $\mathcal{Q}$. If $\varepsilon$ is small enough in this sense, then $\mathcal{P}$ contains a translate $\mathcal{Q}+y$ of $\mathcal{Q}$, and so

$$
\bigcup_{k=1}^{n} \mathcal{Q}+k m x+c_{k}
$$

is $\mathcal{T}$-legal.

If $\mathcal{T}$ has the Meyer property, we will have an "exact" arithmetic progression without the error terms. Assuming that the tiling $\mathcal{T}$ has the Meyer property (page 7), then there is an $r>0$ such that

$$
(\Xi-\Xi) \cap B_{r}=\{0\}
$$

where $\Xi=\Xi(\mathcal{T})$ is the set of all return vectors (page 4). If there is such an $r$, by taking $\varepsilon<r / 4$ we see

$$
(k+1) m x+c_{k+1}-\left(k m x+c_{k}\right)=k m x+c_{k}-\left((k-1) m x+c_{k-1}\right)
$$

for each $k$, and so by denoting this vector by $z$ we see the patch

$$
\bigcup_{k=0}^{n-1} \mathcal{Q}+k z
$$

is $\mathcal{T}$-legal. We have proved the following corollaries: 
Corollary 2.11. If a tiling $\mathcal{T}$ is repetitive and has FLC and the Meyer property, then for any finite $\mathcal{T}$-legal patch $\mathcal{P}$ and $n>0$, there is a $z \in \mathbb{R}^{d} \backslash\{0\}$ such that

$$
\bigcup_{k=1}^{n} \mathcal{P}+k z
$$

is $\mathcal{T}$-legal. The direction $z /\|z\|$ can be chosen arbitrarily close to any given vector of length 1 .

Corollary 2.12. Let $\mathcal{T}$ be a self-affine tiling as in Theorem 2.2 and assume $\operatorname{sp}(Q)$ is a Pisot family. Then for any finite $\mathcal{T}$-legal patch $\mathcal{P}$ and $n>0$, there is a $z \in \mathbb{R}^{d} \backslash\{0\}$ such that the arithmetic progression (7D) is $\mathcal{T}$-legal. The direction $z /\|z\|$ can be chosen to be arbitrarily close to any given vector of length 1.

Recently, this corollary was generalized in [14], as follows:

Theorem 2.13 ([14]). Let $\Lambda$ be a Meyer set in $\mathbb{R}^{d}$. Then, for all positive integers $r$ and $k$, there is an $R>0$ such that, for any coloring of points in $\Lambda$ in $r$ colors and any $x \in \mathbb{R}^{d}$, there is a monochromatic arithmetic progression of length $k$ inside $\Lambda \cap B(x, R)$.

This van der Waerden-type theorem is more general than Corollary 2.12 because it is valid for arbitrary Meyer sets and colorings. Corollary 2.12 is obtained from this theorem, except for the last statement on $z /\|z\|$ in the corollary, when we apply this theorem to the Meyer set $\left\{x \in \mathbb{R}^{d} \mid \mathcal{P}+x \subset \mathcal{T}\right\}$.

Corollary 2.11 proves the existence of arithmetic progressions, given a $\mathcal{P}$ and the length $n$ of the arithmetic progression. Comparing this to Lemma 2.4, we then ask:

Problem 2.14. Take a tiling $\mathcal{T}$, a patch $\mathcal{P}$ and a non-zero return vector $x$. Decide if the arithmetic progression

$$
\bigcup_{k=1}^{n} \mathcal{P}+k x
$$

is always $\mathcal{T}$-legal for every $n>0$.

For a self-affine tiling, we can check all the patterns which can be observed in ball-shaped windows of a certain radius $R>0$. If for some $\mathcal{P}$ and $x$, the arithmetic progressions always stop in the patterns, then we see the arithmetic progressions stop at a fixed finite step anywhere in the tiling. However, we can check this only for a certain choice of $R$ and there are infinite possibilities of $R$. In some cases, we can use induction to show there are arbitrarily long arithmetic progressions (Example 2.7 and Example 2.8) for some $\mathcal{P}$ and $x$. How about the Penrose tilings? We will show that for the Penrose tilings and many self-affine tilings, any arithmetic progression always stops at a fixed finite step, in Section 3.

By the same argument as Corollary 2.12, we can prove the following "full-rank version": 
Theorem 2.15. Let $\mathcal{T}$ be a tiling as in Theorem 2.2 and assume $\operatorname{sp}(Q)$ forms a Pisot family. Then for any finite $\mathcal{T}$-legal patch $\mathcal{P}$ and an $n>0$, there is a basis $\left\{b_{1}, b_{2}, \ldots, b_{d}\right\}$ of $\mathbb{R}^{d}$ such that the patch

$$
\bigcup_{\left(l_{1}, l_{2}, \ldots, l_{d}\right) \in\{1,2, \ldots, n\}^{d}} \mathcal{P}+\sum_{j=1}^{d} l_{j} b_{j}
$$

is $\mathcal{T}$-legal.

The set $\left\{b_{1} /\left\|b_{1}\right\|, b_{2} /\left\|b_{2}\right\|, \ldots, b_{d} /\left\|b_{d}\right\|\right\}$ can be chosen arbitrarily close to any subset of cardinality $d$ of the unit sphere that form a basis of $\mathbb{R}^{d}$, because we can take at least one $\left\{b_{1}, b_{2}, \ldots, b_{d}\right\}$ which forms a basis.

Then the natural question is the following (compare Lemma 2.5) :

Question 2.16. Which tilings $\mathcal{T}$ admit full-rank infinite arithmetic progressions, and which $\mathcal{T}$ do not?

We will address this question for self-affine tilings in Section 4 . Under a certain assumption, the existence of full-rank infinite arithmetic progressions is equivalent to the pure discrete spectrum of the associated tiling dynamical system, in which case we can actually prove that the tiling is limit-periodic.

\section{The non-existence of arbitrarily long arithmetic progres- sions}

In this section, we prove several results on the non-existence of arbitrarily long arithmetic progressions that have a fixed distance, in a self-affine tiling, and so give a partial answer to Problem 2.14.

\subsection{An argument using continuous eigenfunctions}

In this subsection we prove Theorem 3.1, which states that there are not arbitrarily long arithmetic progressions for a fixed difference $v$, if the expansion factor is irrational and Pisot.

Theorem 3.1. Let $\omega$ be a primitive and FLC tiling substitution in $\mathbb{R}^{d}$ with an expansion factor $\lambda$ that is irrational and Pisot. Let $\mathcal{T}$ be a fixed point of $\omega$. Assume $\mathcal{T}$ is repetitive. Then for any $\mathcal{T}$-legal, nonempty and finite patch $\mathcal{P}$ and $v \in \mathbb{R}^{d} \backslash\{0\}$, there is $n_{0} \in \mathbb{N}$ such that $\bigcup_{n=0}^{n_{0}}(\mathcal{P}+n v)$ is not $\mathcal{T}$-legal.

Remark 3.2. If we drop the assumption that $\lambda$ is irrational, there is a counterexample for the statement, as we have shown in Example 2.7. We do not know if there is a counterexample if we drop the assumption that $\lambda$ is Pisot. For the one-dimensional case, there 
is no arbitrarily long arithmetic progressions if $\lambda$ is irrational (Theorem 5.1). Note that $\mathcal{T}$ is non-periodic because the expansion factor is irrational so that the frequency for a tile is irrational.

We divide the proof into several parts, as follows. First we define $A_{\mathcal{P}}$, which will play an important role in the proof.

Definition 3.3. Let $\mathcal{T}$ be a tiling and $\mathcal{P}$ be a patch. We consider a set

$$
A_{\mathcal{P}}=\left\{\mathcal{S} \in X_{\mathcal{T}} \mid \mathcal{P} \subset \mathcal{S}\right\}
$$

Although this is dependent on $X_{\mathcal{T}}$, we denote it just by $A_{\mathcal{P}}$, since in this subsection $\mathcal{T}$ is fixed.

For each $\mathcal{S} \in X_{\mathcal{T}}$, we have $\mathcal{S} \supset \mathcal{P}$ if and only if $\mathcal{S} \in A_{\mathcal{P}}$.

Note that we have defined the notation $\wedge$ in (2) in page 4. In the next lemma, the important point is that we can take $R$ that is independent of $x$.

Lemma 3.4. Let $\mathcal{T}$ be a tiling of $\mathbb{R}^{d}$ which has FLC. Let $f: X_{\mathcal{T}} \rightarrow \mathbb{T}$ be a continuous eigenfunction. Then for any $\varepsilon>0$ there is an $R>0$ such that the conditions

- $\mathcal{S}_{1}, \mathcal{S}_{2} \in X_{\mathcal{T}}$,

- $x \in \mathbb{R}^{d}$, and

- $\mathcal{S}_{1} \wedge B(x, R)=\mathcal{S}_{2} \wedge B(x, R)$

imply $\left|f\left(\mathcal{S}_{1}\right)-f\left(\mathcal{S}_{2}\right)\right|<\varepsilon$. Therefore, given a continuous eigenfunction $f$ and an $\varepsilon>0$, if $R>0$ is large enough and a patch $\mathcal{P}$ covers a ball of radius $R$, then the diameter of $f\left(A_{\mathcal{P}}\right)$ is less than $\varepsilon$.

Proof. Since $f$ is continuous, there is an $R>0$ such that if $\mathcal{S}_{1}, \mathcal{S}_{2} \in X_{\mathcal{T}}$ and

$$
\mathcal{S}_{1} \wedge B(0, R)=\mathcal{S}_{2} \wedge B(0, R),
$$

then $\left|f\left(\mathcal{S}_{1}\right)-f\left(\mathcal{S}_{2}\right)\right|<\varepsilon$. In order to prove the statement of this lemma, take $\mathcal{S}_{1}, \mathcal{S}_{2} \in X_{\mathcal{T}}$ and an $x \in \mathbb{R}^{d}$, and assume

$$
\mathcal{S}_{1} \wedge B(x, R)=\mathcal{S}_{2} \wedge B(x, R)
$$

Then

$$
\left(\mathcal{S}_{1}-x\right) \wedge B(0, R)=\left(\mathcal{S}_{2}-x\right) \wedge B(0, R)
$$

and so $\left|f\left(\mathcal{S}_{1}-x\right)-f\left(\mathcal{S}_{2}-x\right)\right|<\varepsilon$. Since we have

$$
\left|f\left(\mathcal{S}_{1}\right)-f\left(\mathcal{S}_{2}\right)\right|=\left|e^{2 \pi i\langle a, x\rangle} f\left(\mathcal{S}_{1}\right)-e^{2 \pi i\langle a, x\rangle} f\left(\mathcal{S}_{2}\right)\right|=\left|f\left(\mathcal{S}_{1}-x\right)-f\left(\mathcal{S}_{2}-x\right)\right|,
$$


we have proved the first claim.

If $\mathcal{P}$ is a patch that covers $B(x, R)$ for some $x \in \mathbb{R}^{d}$ and $\mathcal{S}_{1}, \mathcal{S}_{2} \in A_{\mathcal{P}}$, then $\mathcal{S}_{1} \wedge B(x, R)=$ $\mathcal{S}_{2} \wedge B(x, R)$, and so $\left|f\left(\mathcal{S}_{1}\right)-f\left(\mathcal{S}_{2}\right)\right|<\varepsilon$. This means that the diameter of $f\left(A_{\mathcal{P}}\right)$ is less than $\varepsilon$.

The proof for Theorem 3.1. Let $\left\{b_{1}, b_{2}, \ldots b_{d}\right\}$ be a basis of $\mathbb{R}^{d}$ consisting of topological eigenvalues for $\left(X_{\mathcal{T}}, \mathbb{R}^{d}\right)$. Such a basis exists by [26, the last part of page 13].

For a $\mathcal{P}$ and a $v$ as in the statement of the theorem, there is an $i$ such that $\left\langle b_{i}, v\right\rangle \neq 0$. Let $f$ be a $\mathbb{T}$-valued continuous eigenfunction for $b_{i}$.

To prove the statement, let us assume on the contrary that for each $n>0$, the patch $\bigcup_{k=0}^{n} \mathcal{P}+k v$ is $\mathcal{T}$-legal. We will show this leads to a contradiction. Since $\omega(\mathcal{T})=\mathcal{T}$, for each $n>0$ and $m>0$, the patch $\mathcal{P}_{n, m}=\bigcup_{k=0}^{n} \omega^{m}(\mathcal{P})+\lambda^{m} k v$ is $\mathcal{T}$-legal, which means that there is an $\mathcal{S}_{n, m} \in X_{\mathcal{T}}$ such that $\mathcal{S}_{n, m} \supset \mathcal{P}_{n, m}$. By the definition of $A_{\omega^{m}(\mathcal{P})}$, for each $n, m>0$ and $k=0,1, \ldots, n$, we have

$$
\mathcal{S}_{n, m}-\lambda^{m} k v \in A_{\omega^{m}(\mathcal{P})} .
$$

This implies that

$$
f\left(\mathcal{S}_{n, m}-\lambda^{m} k v\right)=e^{2 \pi i\left\langle b_{i}, \lambda^{m} k v\right\rangle} f\left(\mathcal{S}_{n, m}\right) \in f\left(A_{\omega^{m}(\mathcal{P})}\right) .
$$

If $m$ is large enough, then the number $\left\langle b_{i}, \lambda^{m} v\right\rangle$ is irrational, and so the diameter of $f\left(A_{\omega^{m}(\mathcal{P})}\right)$ is larger than $1 / 2$. On the other hand, by Lemma 3.4, if $m$ is large enough, then the diameter of $f\left(A_{\omega^{m}(\mathcal{P})}\right)$ can be arbitrarily small. We have a contradiction and have proved the theorem.

Example 3.5. The Penrose tilings are essentially the same (MLD) as the Robinson triangle tilings, which are generated by a substitution with an expansion factor $\frac{1+\sqrt{5}}{2}$. This is an irrational Pisot number and, by Theorem [3.1, we see that there are no arbitrary long arithmetic progressions when we fix $\mathcal{P}$ and $v$, both in the Penrose tilings and the Robinson triangle tilings.

\subsection{An argument using an internal space}

In this subsection, we introduce another method to show the non-existence of arbitrarily long arithmetic progressions in a given self-affine tiling (Theorem 3.6). The argument relies on [18, Theorem 4.1] and is valid for a more general class of self-affine tilings, whereas Theorem 3.1 is valid only for self-similar tilings.

Let $\mathcal{T}$ be a self-affine tiling with an alphabet $\mathcal{A}=\left\{T_{1}, T_{2}, \ldots T_{m}\right\}$, an expansion map $Q$ and a primitive FLC substitution map $\omega$. We assume $Q$ is diagonalizable over $\mathbb{C}$ and all eigenvalues are algebraic conjugates of the same multiplicity $J$. We select control points (page 17) to obtain a Delone set $\Lambda=\bigcup_{i=1}^{m} \Lambda_{i}$, where each $\Lambda_{i}$ is the set of all control points of the tiles of type $i$. 
Since $Q$ is diagonalizable and the multiplicities of the eigenvalues of $Q$ are always $J$, if the real eigenvalues are $\lambda_{1}, \lambda_{2}, \ldots, \lambda_{s}$ and imaginary eigenvalues are $\mu_{1}=a_{1}+b_{1} \sqrt{-1}, \overline{\mu_{1}}=$ $a_{1}-b_{1} \sqrt{-1}, \ldots, \mu_{t}=a_{t}+b_{t} \sqrt{-1}, \overline{\mu_{t}}=a_{t}-b_{t} \sqrt{-1}$, there is a basis $\mathcal{B}$ of $\mathbb{R}^{d}$ such that the matrix $[Q]_{\mathcal{B}}$ of $Q$ with respect to $\mathcal{B}$ is

$$
[Q]_{\mathcal{B}}=\left[\begin{array}{ccccc}
A & 0 & 0 & \cdots & 0 \\
0 & A & 0 & \cdots & 0 \\
\vdots & & \ddots & & \vdots \\
0 & \cdots & & & A
\end{array}\right]
$$

where

$$
A=\left[\begin{array}{ccccccccc}
\lambda_{1} & 0 & 0 & \ldots & & & & & 0 \\
0 & \lambda_{2} & 0 & \ldots & & & & & 0 \\
\vdots & & \ddots & & & & & & 0 \\
& & & \lambda_{s} & & & & & \\
& & & & a_{1} & -b_{1} & \cdots & & \\
& & & & b_{1} & a_{1} & & & \\
& & & & & & \ddots & & \\
0 & & & & & & & a_{t} & -b_{t} \\
0 & & & & & & & b_{t} & a_{t}
\end{array}\right] .
$$

(This is the real canonical form of $Q$.) Let $\alpha_{j}(j=1,2, \ldots, J)$ be the vector of $\mathbb{R}^{d}$ such that the representation with respect to the basis $\mathcal{B}$ is

$$
(0,0, \ldots, 0, \underbrace{1,1, \ldots 1}_{\text {from }(j-1) m+1 \text { to } j m} \quad 0, \ldots 0)
$$

where $m=s+2 t$.

By [18, Theorem 4.1], there exists an isomorphism $\rho: \mathbb{R}^{d} \rightarrow \mathbb{R}^{d}$ such that $\rho Q=Q \rho$ and

$$
\Lambda \subset \rho\left(\mathbb{Z}[Q] \alpha_{1}+\cdots+\mathbb{Z}[Q] \alpha_{J}\right)
$$

This is the first tool that we use in Theorem 3.6.

The other tool is the following. By [27, Lemma 6.5], there exists a finite set $F \subset \Lambda-\Lambda$ such that for any element $x \in \Lambda-\Lambda$, there are an $n>0$ and $w_{1}, w_{2}, \ldots, w_{n} \in F$ with

$$
x=\sum_{i=0}^{n} Q^{i} w_{i} .
$$


In fact, $x=c(T)-c(S)$ for some tiles $T$ and $S$ in $\mathcal{T}$. By repetitivity, we may assume that $T$ and $S$ are both in $\omega^{n}(U)$ for some $n>0$ and $U \in \mathcal{T}$. There are $T^{(0)}=U, T^{(1)} \in$ $\omega\left(T^{(0)}\right), T^{(2)} \in \omega\left(T^{(1)}\right), \ldots$ such that $T=T^{(n)} \in \omega\left(T^{(n-1)}\right)$. We have

$$
c(T)=\sum_{k=0}^{n-1} Q^{n-1-k}\left(c\left(T^{(k+1)}\right)\right)-Q^{n-k}\left(c\left(T^{(k)}\right)\right)+Q^{n}\left(c\left(T^{(0)}\right)\right) .
$$

There are also $S^{(0)}=U, S^{(1)} \in \omega\left(S^{(0)}\right), S^{(2)} \in \omega\left(S^{(1)}\right), \ldots$ such that $S=S^{(n)} \in \omega\left(S^{(n-1)}\right)$. We have

$$
c(S)=\sum_{k=0}^{n-1} Q^{n-1-k}\left(c\left(S^{(k+1)}\right)\right)-Q^{n-k}\left(c\left(S^{(k)}\right)\right)+Q^{n}\left(c\left(S^{(0)}\right)\right) .
$$

Therefore,

$$
c(T)-c(S)=\sum_{k=0}^{n-1} Q^{n-1-k}\left(c\left(T^{(k+1)}\right)-Q\left(c\left(T^{(k)}\right)\right)\right)-\sum_{k=0}^{n-1} Q^{n-1-k}\left(c\left(S^{(k+1)}\right)-Q\left(c\left(S^{(k)}\right)\right)\right) .
$$

Each of $c\left(T^{(k+1)}\right)-Q\left(c\left(T^{(k)}\right)\right)$ and $c\left(S^{(k+1)}\right)-Q\left(c\left(S^{(k)}\right)\right)$ is a difference of tiles inside a level-one supertile.

With these machinery, we can prove the following theorem. The idea is to use a kind of internal space. For a model set $D$ [3, Chapter 7] with a Euclidean internal space, if there are arbitrarily long arithmetic progressions $x, x+y, x+2 y, \ldots x+n y \in D$ with a fixed $y \neq 0$, then since $y^{*} \neq 0$, for a large $n$, the corresponding point $x^{*}+n y^{*}$ should be outside the window and we have a contradiction. Here, we construct a similar Euclidean "internal space" and have a similar argument to obtain a contradiction if there are arbitrarily long arithmetic progressions.

Theorem 3.6. Let $\mathcal{T}$ be a self-affine tiling of $\mathbb{R}^{d}$ with an expansion map $Q$ and a primitive $F L C$ substitution rule $\omega$. Suppose $Q$ is diagonalizable over $\mathbb{C}$ and all the eigenvalues are algebraic conjugates with the same multiplicity $J$. If there exists an algebraic conjugate $\beta$ of eigenvalues of $Q$ such that $|\beta|<1$, then for any return vector $x \in \mathbb{R} \backslash\{0\}$, there is an $n_{0} \in \mathbb{N}$ such that $T+n x \notin \mathcal{T}$ for all $n>n_{0}$ and $T \in \mathcal{T}$. Therefore, for each $T \in \mathcal{T}$ and $x \neq 0$, there is an $n_{0}>0$ such that $\bigcup_{k=1}^{n_{0}} T+k x$ is not $\mathcal{T}$-legal.

Proof. Without loss of generality, we may assume that $\rho$ is the identity.

For each $q_{1}(x), q_{2}(x), \cdots q_{J}(x) \in \mathbb{Z}[x]$, the representation of the vector $q_{1}(Q) \alpha_{1}+\cdots+$ $q_{J}(Q) \alpha_{J}$ with respect to the basis $\mathcal{B}$ is

$$
\left[\begin{array}{c}
q_{1}(A) \mathbf{1} \\
q_{2}(A) \mathbf{1} \\
\vdots \\
q_{J}(A) \mathbf{1}
\end{array}\right]
$$


where $\mathbf{1} \in \mathbb{R}^{m}$ is the vector whose entries are all 1. Define a well-defined injective map $\Phi: \mathbb{Z}[Q] \alpha_{1}+\cdots+\mathbb{Z}[Q] \alpha_{J} \rightarrow \mathbb{C}^{J}$ by

$$
\left[\begin{array}{c}
q_{1}(A) \mathbf{1} \\
q_{2}(A) \mathbf{1} \\
\vdots \\
q_{J}(A) \mathbf{1}
\end{array}\right] \mapsto\left[\begin{array}{c}
q_{1}(\beta) \\
q_{2}(\beta) \\
\vdots \\
q_{J}(\beta)
\end{array}\right]
$$

Since the set $F$ in (9) is finite and $|\beta|<1$, we can observe that the image of $\Lambda$ by $\Phi$ is bounded. If $x \in \Lambda-\Lambda$ and $x \neq 0$, then since $\Phi(x) \neq 0$, there is an $n_{0}$ such that $\left\|\Phi\left(n_{0} x\right)\right\|=\left\|n_{0} \Phi(x)\right\|$ is greater than the diameter of $\Phi(\Lambda)$. This implies that if $y \in \Lambda$, then for each $n>n_{0}$, we have $\Phi(y+n x) \notin \Phi(\Lambda)$ and $y+n x \notin \Lambda$.

\section{The existence and non-existence of full-rank infinite arith- metic progressions}

In this section, we investigate Question 2.16 for self-affine tilings in $\mathbb{R}^{d}$. In particular, we prove that, under additional assumptions, the existence of full-rank infinite arithmetic progressions implies the pure discrete spectrum of the tiling (Theorem 4.7), and the converse (Corollary 4.11, Corollary 4.14, Theorem 4.15). Then the conclusion is strengthened by showing that the tiling is in fact limit periodic (Theorem 4.18, Corollary 4.19).

\subsection{Full-rank infinite arithmetic progressions imply pure discrete spec- trum}

The following setting is assumed throughout the subsection.

Setting 1. In this subsection, $\mathcal{T}$ is a self-affine tiling with an expansion map $Q$ and a primitive FLC substitution map $\omega$.

The key notion here is the overlap coincidence, which is under an mild assumption equivalent to pure discrete spectrum.

Definition $4.1([27])$. A triple $(T, y, S)$, with $T, S \in \mathcal{T}$ and $y \in \Xi(\mathcal{T})$, is called an overlap if the intersection $\operatorname{supp}(y+T) \cap \operatorname{supp}(S)$ has a non-empty interior. We say that two overlaps $(T, y, S)$ and $\left(T^{\prime}, y^{\prime}, S^{\prime}\right)$ are equivalent if for some $g \in \mathbb{R}^{d}$ we have $y+T=g+y^{\prime}+T^{\prime}, S=$ $g+S^{\prime}$. Denote by $[(T, y, S)]$ the equivalence class of an overlap. An overlap $(T, y, S)$ is a coincidence if $y+T=S$. The support of an overlap $(T, y, S)$ is $\operatorname{supp}(T, y, S)=$ $\operatorname{supp}(y+T) \cap \operatorname{supp}(S)$.

We define the subdivision graph $\mathcal{G}_{\mathcal{O}}(\mathcal{T})$ for overlaps. Its vertices are the equivalence classes of overlaps for $\mathcal{T}$. Let $\mathcal{O}=(T, y, S)$ be an overlap. We will specify directed edges 
leading from the equivalence class $[\mathcal{O}] . \omega(y+T)=Q y+\omega(T)$ is a patch of $Q y+\mathcal{T}$, and $\omega(S)$ is a $\mathcal{T}$-patch, and moreover,

$$
\operatorname{supp}(Q y+\omega(T)) \cap \operatorname{supp}(\omega(S))=Q(\operatorname{supp}(T, y, S))
$$

For each pair of tiles $T^{\prime} \in \omega(T)$ and $S^{\prime} \in \omega(S)$ such that $\mathcal{O}^{\prime}:=\left(T^{\prime}, Q y, S^{\prime}\right)$ is an overlap, we draw a directed edge from $[\mathcal{O}]$ to $\left[\mathcal{O}^{\prime}\right]$.

The following equivalence is useful when we discuss overlaps.

Lemma 4.2. For an overlap $\mathcal{O}=(T, y, S)$, the following conditions are equivalent:

1. there is an $n>0$ such that $\omega^{n}(T+y) \cap \omega^{n}(S) \neq \emptyset$;

2. from the equivalence class $[\mathcal{O}]$ there is a path that leads to the equivalence class of a coincidence.

We also use the following two lemmas concerning overlaps.

Lemma 4.3 ([19], Lemma A.8). Assume that $\Xi(\mathcal{T})$ is a Meyer set. Then the number of equivalence classes of overlaps for $\mathcal{T}$ is finite.

We set

$$
D_{x}=\mathcal{T} \cap(\mathcal{T}+x)
$$

for a vector $x \in \mathbb{R}^{d}$.

The following lemma is slightly different from [19, Lemma A.9], but the proof is the same.

Lemma 4.4 ([19], Lemma A.9). Assume that $\Xi(\mathcal{T})$ is a Meyer set. Let $x \in \Xi(\mathcal{T})$. The following are equivalent:

(i) $\lim _{n \rightarrow \infty} \operatorname{dens}\left(D_{Q^{n} x}\right)=1$;

(ii) $1-\operatorname{dens}\left(D_{Q^{n} x}\right) \leq C r^{n}, n \geq 1$, for some $C>0$ and $r \in(0,1)$;

(iii) From each vertex $\mathcal{O}$ of the graph $\mathcal{G}_{\mathcal{O}}(\mathcal{T})$ such that $\mathcal{O}=\left[\left(T, Q^{k} x, S\right)\right]$ for some $T, S \in \mathcal{T}$ and $k>0$, there is a path leading to a coincidence.

Theorem 4.5 ([27], Theorem 6.1). Assume that $\Xi(\mathcal{T})$ is a Meyer set. If there exists a basis $\mathcal{B}$ for $\mathbb{R}^{d}$ such that for all $x \in \mathcal{B}$,

$$
\sum_{n=0}^{\infty}\left(1-\operatorname{dens}\left(D_{Q^{n} x}\right)\right)<\infty,
$$

then the tiling dynamical system $\left(X_{\mathcal{T}}, \mu, \mathbb{R}^{d}\right)$ has pure discrete spectrum.

By these two results we have the following corollary: 
Corollary 4.6. Assume that $\Xi(\mathcal{T})$ is a Meyer set. Let $\mathcal{B}$ be a basis of $\mathbb{R}^{d}$ that is included in $\Xi(\mathcal{T})$. Suppose that for each overlap $\left(S, Q^{k} y, T\right)$ with $y \in \mathcal{B}$ and $k>0$, there is a path from $\left[\left(S, Q^{k} y, T\right)\right]$ to a coincidence (that is, there is an $n>0$ such that $\left.\omega^{n}\left(S+Q^{k} y\right) \cap \omega^{n}(T) \neq \emptyset\right)$. Then the dynamical system $\left(X_{\mathcal{T}}, \mu, \mathbb{R}^{d}\right)$ is pure discrete.

Theorem 4.7. Suppose that there exist a tile $T_{0} \in \mathcal{T}$ and a lattice $L$ on $\mathbb{R}^{d}$ such that $\left\{T_{0}+v \mid v \in L\right\} \subset \mathcal{T}$ and $Q(L) \subset L$. Then $\left(X_{\mathcal{T}}, \mu, \mathbb{R}^{d}\right)$ has pure discrete spectrum.

Proof. Note that $\Xi(\mathcal{T})$ is a Meyer set by the existence of a full-rank infinite arithmetic progression and FLC: any return vector is written as the sum of the displacement to the nearest tile in a full-rank infinite arithmetic progression, a vector inside $L$, and the displacement of a tile in a full-rank infinite arithmetic progression to the target. We also note that $L \subset \Xi(\mathcal{T})$. We consider the overlaps which can occur from the translation vectors in $L$. For each such overlap $\mathcal{O}=(T, y, S), y \in L$, we can find some integer $n \in \mathbb{N}$ such that the $n$-th inflation of the support of the overlap $\mathcal{O}$ contains at least one element of $\left\{T_{0}+v \mid v \in L\right\}$, i.e. there exists $u \in L$ such that

$$
\operatorname{supp}\left(T_{0}+u\right) \subset Q^{n}(\operatorname{supp}(T, y, S)) .
$$

By the assumption that $\left\{T_{0}+v \mid v \in L\right\} \subset \mathcal{T}$,

$$
T_{0}+u \in \omega^{n}(S) .
$$

Since $Q L \subset L, T_{0}+u-Q^{n} y \in \omega^{n}(T)$. Therefore, the overlap $\mathcal{O}=(T, y, S)$ admits a coincidence in the $n$-th iteration. We can choose a set of the vectors $\mathcal{B}:=\left\{y_{1}, \ldots, y_{d}\right\} \subset L$ which forms a basis of $\mathbb{R}^{d}$. By using $Q \mathcal{B} \subset L$ and applying Corollary 4.6 to $\mathcal{B}$, we see the dynamical system is pure discrete.

Example 4.8. The condition $Q(L) \subset L$ is always satisfied if $Q=n I$ for some natural number $n$. The table tilings (Example 2.8) do not admit full-rank infinite arithmetic progressions.

\subsection{Pure discrete spectrum and a lattice property imply full-rank infinite arithmetic progressions}

In this subsection, we discuss the existence of full-rank infinite arithmetic progressions under the following setting:

Setting 2. Let $\omega$ be a primitive $F L C$ substitution rule in $\mathbb{R}^{d}$ with an expansion map $Q$. Let us denote the alphabet by $\mathcal{A}=\left\{T_{1}, T_{2}, \ldots, T_{m}\right\}$. Let $\mathcal{T}$ be a self-affine tiling generated by $\omega$. We take a control point from each tile of $\mathcal{T}$. The symbol $\Lambda_{i}$ will denote the set of all control points of tiles of type $i$. Let $\Xi=\Xi(\mathcal{T})$ be the set of all return vectors of $\mathcal{T}$. 
In this subsection, we first prove sufficient conditions for $\mathcal{T}$ to admit full-rank infinite arithmetic progressions. We use the following characterization of having pure discrete dynamical spectrum:

Theorem 4.9 ([15], Theorem 3.13). Let $\mathcal{S}$ be a self-affine tiling with an expansion map $Q$ and an alphabet $\mathcal{B}$. We take control points for the tiles in $\mathcal{S}$, and let $M_{T}$ be the set of all control points of type $T \in \mathcal{B}$. Then the following are equivalent:

1. $\mathcal{S}$ has pure discrete dynamical spectrum;

2. There are an $N>0$, an $S \in \mathcal{B}$ and an $\eta \in M_{S}$ such that

$$
Q^{N}(\Xi(\mathcal{S})) \subset M_{S}-\eta
$$

where $\Xi(\mathcal{S})$ is the set of all return vectors for $\mathcal{S}$.

The second condition in this characterization is called the algebraic coincidence. Using algebraic coincidences, we now prove the following.

Corollary 4.10. Let $L$ be a discrete subgroup of $\mathbb{R}^{d}$. Consider the following four conditions:

1. there are a positive integer $K$ and a $T \in \mathcal{T}$ such that $\left\{T+x \mid x \in Q^{K}(L)\right\} \subset \mathcal{T}$;

2. $\Xi \supset Q^{K}(L)$ for some positive integer $K$;

3. there is a positive integer $K$ such that, for each positive integer $k$, we have

$$
\underbrace{\Xi+\Xi+\cdots+\Xi}_{k \text { times }} \supset Q^{K}(L)
$$

4. there are positive integers $k$ and $K$ such that (11) holds.

Then we always have $1 . \Rightarrow 2 . \Rightarrow 3 . \Rightarrow 4$., and if $\mathcal{T}$ have pure discrete dynamical spectrum, we have $4 . \Rightarrow 1$.

Proof. The implication 1. $\Rightarrow 2$. is proved by the definition of $\Xi$, the implication 2 . $\Rightarrow 3$. is seen by the fact that $0 \in \Xi$ and the implication 3 . $\Rightarrow 4$. is clear. Let us now assume the condition 4 . and that $\mathcal{T}$ has pure discrete dynamical spectrum.

By the algebraic coincidence, we have

$$
Q^{2 N}(\Xi+\Xi) \subset Q^{N}\left(\Lambda_{i}-\Lambda_{i}\right) \subset Q^{N}(\Xi)
$$

By induction, we see

$$
Q^{k N+K}(L) \subset Q^{k N}(\underbrace{\Xi+\Xi+\cdots+\Xi}_{k \text { times }}) \subset Q^{N}(\Xi) \subset \Lambda_{i}-\eta
$$

This implies that $\left\{T_{i}+\eta+x \mid x \in Q^{k N+K}(L)\right\} \subset \mathcal{T}$. 
By this theorem, if $\mathcal{T}$ has pure discrete dynamical spectrum, the question of whether $\mathcal{T}$ admits arithmetic progressions boils down to deciding if the conditions 2.,3., or 4. hold. We first discuss the easiest case where these conditions are satisfied.

Corollary 4.11. Assume that the group L generated by $\Xi$ is a lattice in $\mathbb{R}^{d}$ and that the tiling $\mathcal{T}$ has pure discrete dynamical spectrum. Then there are a positive integer $K$ and $T \in \mathcal{T}$ such that $\left\{T+x \mid x \in Q^{K}(L)\right\} \subset \mathcal{T}$. In other words, $\mathcal{T}$ admits a full-rank infinite arithmetic progression.

Proof. Since $\Xi$ is relatively dense in $\mathbb{R}^{d}$, we may take an $R>0$ such that $\Xi+B_{R}=\mathbb{R}^{d}$. The set $L \cap B_{R}$ is a finite set and so there is a positive integer $M$ such that

$$
L \cap B_{R} \subset \underbrace{\Xi+\Xi+\cdots+\Xi}_{M \text { times }} .
$$

For each $x \in L$, there is a $y \in \Xi$ such that $\|x-y\| \leq R$. Since $x-y \in L \cap B_{R}$, we see

$$
x=y+x-y \in \underbrace{\Xi+\Xi+\cdots+\Xi}_{M+1 \text { times }} .
$$

We conclude that

$$
L=\underbrace{\Xi+\Xi+\cdots+\Xi}_{M+1 \text { times }} \text {. }
$$

The equivalent conditions in Corollary 4.10 are satisfied.

Example 4.12. Suppose $\omega$ is a block substitution (a block inflation in [21, Subsection $5.2]$ ). This is a substitution rule where the supports of the proto-tiles are all $[0,1]^{d}$ and the map $Q$ is given by a diagonal matrix with integer diagonal elements. The group generated by $\Xi$ is always a lattice. $\mathcal{T}$ admits full-rank infinite arithmetic progressions if it has pure discrete dynamical spectrum.

We next give a general sufficient condition for the condition 4. in Corollary 4.10. The proof is given in Appendix.

Proposition 4.13. Let $1 \leqq e \leqq d$ and $v_{1}, v_{2}, \ldots, v_{e}$ be vectors in $\mathbb{R}^{d}$. Assume the following conditions:

1. for each $i=1,2, \ldots, e$, there is a positive integer $n_{i}$ such that $Q v_{i}=n_{i} v_{i}$, and

2. there are $T^{(0)} \in \mathcal{T}, T_{1}^{(0)}, T_{2}^{(0)}, \ldots, T_{e}^{(0)} \in \omega\left(T^{(0)}\right)$ of the same tile type such that

$$
c\left(T_{i}^{(0)}\right)-Q\left(c\left(T^{(0)}\right)\right)=v_{i}
$$

for each $i$. 
Then there is a positive integer $M$ such that we have

$$
\operatorname{span}_{\mathbb{Z}}\left\{v_{1}, v_{2}, \ldots, v_{e}\right\} \subset \underbrace{\Xi+\Xi+\cdots+\Xi}_{M \text { times }} \text {. }
$$

Corollary 4.14. Let $e \leqq d$ and $v_{1}, v_{2}, \ldots, v_{e}$ be vectors in $\mathbb{R}^{d}$ that form a linearly independent set. Denote the group generated by $\left\{v_{1}, v_{2}, \ldots, v_{e}\right\}$ by L. If the two assumptions in Proposition 4.13 is satisfied and $\mathcal{T}$ has pure discrete dynamical spectrum, then there is a tile $T \in \mathcal{T}$ and a positive integer $K$ such that $\left\{T+x \mid x \in Q^{K}(L)\right\} \subset \mathcal{T}$.

Proof. The claim follows from Corollary 4.10 and Proposition 4.13

For the self-similar case, the situation is simple:

Theorem 4.15. Assume $Q=n I$, where $n$ is a positive integer and $I$ is the identity map. Then there are a lattice $L$ of $\mathbb{R}^{d}$ and a positive integer $M$ such that

$$
L \subset \underbrace{\Xi+\Xi+\cdots+\Xi}_{M \text { times }} \text {. }
$$

If $\mathcal{T}$ has pure discrete dynamical spectrum, then there are also a positive integer $K$ and a $T \in \mathcal{T}$ such that

$$
\left\{T+x \mid x \in n^{K} L\right\} \subset \mathcal{T} \text {. }
$$

In other words, $\mathcal{T}$ admits a full-rank infinite arithmetic progression.

Proof. We give two proofs and the first one relies on Proposition 4.13. By replacing $Q$ and $\omega$ with their powers, by repetitivity we may take a $T^{(0)} \in \mathcal{T}$, a linearly independent $\left\{v_{1}, v_{2}, \ldots, v_{d}\right\} \subset \mathbb{R}^{d}$ and $T_{1}^{(0)}, T_{2}^{(0)}, \ldots T_{d}^{(0)} \in \omega\left(T^{(0)}\right)$ such that

- $T_{1}^{(0)}, T_{2}^{(0)}, \ldots T_{d}^{(0)}$ are the same tile type as $T^{(0)}$, and

- $c\left(T_{i}^{(0)}\right)-Q\left(c\left(T^{(0)}\right)\right)=v_{i}$ for each $i$.

The first statement of this theorem is proved by Proposition 4.13. The second statement is seen by Corollary 4.10 .

The second proof does not use Proposition 4.13 and relies on a result by Kenyon, which is found in [26, Theorem 5.1], or its generalization [16, Theorem 4.1]. There is a basis $\left\{b_{1}, b_{2}, \ldots, b_{d}\right\}$ of $\mathbb{R}^{d}$ such that

$$
\Xi \subset \mathbb{Z} b_{1}+\mathbb{Z} b_{2}+\cdots+\mathbb{Z} b_{d}
$$

The group $L$ generated by $\Xi$ is a lattice of $\mathbb{R}^{d}$. By the same argument as Corollary 4.11, the first statement is proved. The second statement follows from Corollary 4.10 , 
We will show that for irrational expansion factors, there are no full-rank infinite arithmetic progressions in self-similar tilings (Theorem 5.1).

In fact, we can prove a stronger statement that $\mathcal{T}$ is limit-periodic, which is defined as follows. To define it, recall the densities of patches are defined in (3) in page 4. Using densities, we define the limit periodic tilings:

Definition 4.16 ([7], Definition 4.2). We say that a tiling $\mathcal{S}$ is limit periodic if there are a subset $\mathcal{P} \subset \mathcal{S}$ of zero density and a decreasing sequence $L_{1} \supset L_{2} \supset \cdots$ of lattices of $\mathbb{R}^{d}$ such that for any $T \in \mathcal{S} \backslash \mathcal{P}$ there is $n$ with $\left\{T+x \mid x \in L_{n}\right\} \subset \mathcal{S}$.

For example, the period doubling sequence and the repetitive fixed points of the chair substitution are limit periodic ([5]).

Remark 4.17. It is hard to find the definition of limit-periodic tilings in the literature and different authors seem to use the term for different meanings. Limit-periodic tilings are geometric analogues for Toeplitz sequences [13]. The regular Toeplitz sequences have pure discrete dynamical spectrum, but there are non-regular Toeplitz sequences with non-zero continuous spectrum [12, which means a "Toeplitz structure" in a definition of limitperiodic tilings does not imply pure discrete dynamical spectrum. The definition of limitperiodicity in [4] seems to include pure discrete dynamical spectrum, whereas in [9] the authors do not include it. Here, we adopt the definition in [7] and do not assume pure discrete spectrum. Note that by Theorem 4.7, if $\mathcal{T}$ is self-similar with $Q=n I$, where $n$ is a positive integer and $I$ is the identity map, the limit periodicity of $\mathcal{T}$ implies the pure discrete dynamical spectrum.

We now have the following theorem. Note that we assumed the conditions in Setting 2 at the beginning of this subsection.

Theorem 4.18. If $\mathcal{T}$ has pure discrete dynamical spectrum and satisfies the equivalent conditions in Corollary 4.10, then the tiling $\mathcal{T}$ is limit periodic. In particular, there is a lattice $L$ and a patch $\mathcal{P} \subset \mathcal{T}$ of zero density such that, if $T \in \mathcal{T} \backslash \mathcal{P}$, then $\{T+x \mid x \in$ $\left.Q^{l}(L)\right\} \subset \mathcal{T}$ for some natural number $l$.

Proof. The proof is given in Appendix, and here, we shall give an intuitive explanation of the idea of the proof. We recall the set $D_{x}=\mathcal{T} \cap(\mathcal{T}-x)$ in (10). For each $k=1,2, \ldots$, consider the set

$$
\bigcap_{x \in \Xi} D_{Q^{k} x}
$$

If $T$ is in this set, then for all $x \in \Xi$, we have $T \in \mathcal{T}-Q^{k}(x)$. By assumption, there is an $M>0$ such that $Q^{M}(L) \subset \Xi$, and so for each $x \in L$, we have $T+Q^{k+M}(x) \in \mathcal{T}$. In other words, $T$ is a part of a full-rank infinite arithmetic progression. If the density of the set (13) is close to 1 , then there are "many" $T$ in this set, and each of such $T$ satisfies the 
condition $\left\{T+Q^{M+k}(x) \mid x \in L\right\} \subset \mathcal{T}$. Thus it suffices to show that the set (13) has large density. More precisely, it suffices to show the density of (13) tends to 1 as $k \rightarrow \infty$.

This is seen by modifying the proof for the claim that an overlap coincidence implies an algebraic coincidence [15]. The set (13) is written as

$$
\bigcap_{x \in \Xi} D_{Q^{k}(x)}=\bigcup_{T \in \mathcal{T}} \bigcap_{x \in \Xi} \omega^{k}(T) \cap \omega^{k}(\mathcal{T}-x) .
$$

In other words, for each $T \in \mathcal{T}$, we can consider all possible overlaps $(T, x, S)$. By inflation, it gives rise to coincidences and the set of all coincidences for this overlap by the $k$ th inflation is $\omega^{k}(T) \cap \omega^{k}(S-x)$. Inside the supertile $\omega^{k}(T)$, there are other sets of coincidences $\omega^{k}(T) \cap \omega^{k}\left(S^{\prime}-x\right)$ with the same $x$ but a different tile $S^{\prime}$. Fixing $x$, we collect all such coincidences, and the resulting set is $\omega^{k}(T) \cap \omega^{k}(\mathcal{T}-x)$. The ratio of the area that this set of coincidences covers to the area that the supertile $\omega^{k}(T)$ covers tends to 1 , by the argument of Lemma 4.4. Moreover, since there are only finitely many overlaps, the intersection

$$
\bigcap_{x \in \Xi} \omega^{k}(T) \cap \omega^{k}(\mathcal{T}-x)
$$

is in fact a finite intersection. For each $x \in \Xi$, the set $\omega^{k}(T) \cap \omega^{k}(\mathcal{T}-x)$ grows, and so the ratio of the area that the patch (14) covers to the support $\operatorname{supp} \omega^{k}(T)$ tends to 1 .

This happens for each $T \in \mathcal{T}$, and again by using the fact that there are only finitely many overlaps up to equivalence, the convergence of the ratio of the area of (14) to 1 is uniform for all $T \in \mathcal{T}$. This in turn implies that the density of (13) tends to 1 .

Corollary 4.19. Suppose that the tiling $\mathcal{T}$ has pure discrete dynamical spectrum. Moreover, assume one of the following conditions:

1. the group generated by $\Xi$ is a lattice;

2. there is a basis $\left\{b_{1}, b_{2}, \ldots, b_{d}\right\}$ of $\mathbb{R}^{d}$ that satisfies the two assumptions in Proposition 4.13;

3. there is a positive integer $n$ such that $Q=n I$.

Then $\mathcal{T}$ is limit-periodic.

Proof. By using Theorem 4.18, this follows from Corollary 4.11, Proposition 4.13 and Theorem 4.15,

Example 4.20. The block substitution (Example 4.12) satisfies the first condition in Corollary 4.19, If the substitution satisfies the coincidence condition (a high-dimensional version of Dekking's condition [25, Definition 6.5], which follows from the overlap algorithm [27]), then it is limit-periodic. 
The self-similar tiling by the sphinx substitution [10] are known to be limit-periodic. We give another proof for this fact: we can check that the sphinx substitution satisfies the overlap coincidence [27, Example 7.2]; by Corollary 4.19, the fact that the expansion factor is 2 implies the sphinx tilings are limit-periodic.

\section{The conclusion for full-rank infinite arithmetic progres- sions in self-similar tilings}

By what we have proved, we have the following complete picture for the existence/nonexistence of full-rank infinite arithmetic progressions in any self-similar tiling:

Theorem 5.1. Let $\mathcal{T}$ be a self-similar tiling in $\mathbb{R}^{d}$ with a primitive FLC substitution and an expansion factor $\lambda$.

1. If $\lambda$ is irrational, then there are no full-rank infinite arithmetic progressions in $\mathcal{T}$.

2. If $\lambda$ is rational (i.e. it is a natural number), then the following three conditions are equivalent:

(a) $\mathcal{T}$ admits full-rank infinite arithmetic progressions;

(b) $\mathcal{T}$ is limit periodic;

(c) $\mathcal{T}$ has pure discrete dynamical spectrum.

Proof. The second statement is clear by Theorem 4.7 and Corollary 4.19, We shall prove the first statement.

Suppose that $\lambda$ is irrational. The statement follows from the observation that if we have two full-rank infinite arithmetic progressions with incommensurate distances, then there are two different tiles that are arbitrarily close, which contradicts the fact that $\mathcal{T}$ is a tiling. We shall elaborate the argument. We assume there are a $T \in \mathcal{T}$ and a lattice $L$ of $\mathbb{R}^{d}$ such that $\{T+x \mid x \in L\} \subset \mathcal{T}$ and we will obtain a contradiction. We take a basis $\left\{b_{1}, b_{2}, \ldots, b_{d}\right\}$ of $\mathbb{R}^{d}$ such that $L$ is the $\mathbb{Z}$-span of this basis.

We can take a natural number $N$ such that there is a $y \in \mathbb{R}^{d}$ with $T+y \in \omega^{N}(T)$. For each $\varepsilon>0$, we can take integers $n_{j}, m_{j}(j=1,2, \ldots, d)$, such that

$$
0<\left\|\lambda^{N} \sum_{j=1}^{d} n_{j} b_{j}+y-\sum_{j=1}^{d} m_{j} b_{j}\right\|<\varepsilon .
$$

These inequalities show that the two tiles $T+\lambda^{N} \sum n_{j} b_{j}+y$ and $T+\sum m_{j} b_{j}$ are different but their interiors overlap. Since these tiles are both in $\mathcal{T}$, we obtain a contradiction. 
Remark 5.2. Although we described Theorem 5.1 as a "complete picture", it is not clear when $\mathcal{T}$ has pure discrete dynamical spectrum. The Pisot conjecture, which states a selfsimilar tiling for any irreducible substitution with Pisot expansion factor has pure discrete dynamical spectrum, has not been proved. However, we have an algorithm to decide if a given self-similar tiling has pure discrete dynamical spectrum ([1, 27]).

For the one-dimensional case, if the substitution $\omega$ is also irreducible, we can prove the first claim of Theorem 5.1 by using the Perron-Frobenius theory, as follows.

Assume that $\omega$ has an indexed alphabet $\mathcal{A}=\left\{T_{1}, T_{2}, \ldots, T_{m}\right\}$ and is primitive. Assume also that the support of each tile in $\mathcal{A}$ is an interval. By the Perron-Frobenius theorem, there is a positive eigenvalue that is greater than any other eigenvalues in modulus. We call it the Perron-Frobenius eigenvalue, and for substitutions it coincides with the expansion factor. Let $\left(l_{j}\right)_{j=1,2, \ldots, m}$ and $\left(r_{j}\right)_{j=1,2, \ldots, m}$ be left and right eigenvectors, respectively, with positive elements, which exist uniquely up to scalar multiples.

Lemma 5.3. [2, Lemma 4.3] If $\omega$ is primitive and irreducible, then the d-entries of $\left(l_{j}\right)_{j \in A}$ $\left(\right.$ resp. $\left.\left(r_{j}\right)_{j \in A}\right)$ are linearly independent over $\mathbb{Q}$.

Recall that by primitivity, the corresponding tiling dynamical system is uniquely ergodic, which is equivalent to having uniform patch frequency.

We now prove that an infinite arithmetic progression cannot be observed in a selfsimilar tiling generated by an irreducible primitive substitution $\omega$ with the cardinality of the alphabet greater than 1 . Note that the irreducibility implies that $\lambda$ is irrational.

Theorem 5.4. Let $\omega$ be a one-dimensional irreducible primitive substitution with interval supports of the tiles in the alphabet and with the cardinality of the alphabet greater than 1. Let $\mathcal{T}_{0}$ be a repetitive fixed point for $\omega$. If $\mathcal{T}$ is a tiling in $X_{\mathcal{T}_{0}}$, then for any tile $T \in \mathcal{T}$ and non-zero vector $v$, the patch $\{T+k v \mid k \in \mathbb{N}\}$ is not included in $\mathcal{T}$. Therefore, there are no arbitrarily long arithmetic progressions if we fix the distance $v \neq 0$.

Proof. Assume that $\{T+k v \mid k \in \mathbb{N}\}$ is included in $\mathcal{T}$. We prove that this leads to a contradiction, for the case where $v>0$. The case where $v<0$ is proved in a similar way. Consider a legal patch $\mathcal{P}_{k}(k \in \mathbb{N})$ of interval support whose left most tile is $T+k v$ and right most tile is the tile just before $T+(k+1) v$. The support of the patch $\mathcal{P}_{k}$ is an interval of the fixed length $v$ which is filled by translates of alphabets $T_{j}$ 's with cardinalities $n_{j}$ 's where $j \in\{1,2, \ldots n\}$. Then the cardinality vector $\left(n_{j}\right)_{j \in\{1,2, \ldots n\}} \in \mathbb{N}^{d}$ is independent of the choice of $k$ by Lemma 5.3. For each $m>0$, the patch $\bigcup_{k=0}^{m-1} \mathcal{P}_{k}$ contains $m n_{j}$ translates of $T_{j}$ for $j \in\{1,2, \ldots, n\}$ and has support $[0, m v]$. By the uniform patch frequency, this shows that $r_{i}=n_{i} /\left(\sum_{j=1}^{n} n_{j}\right) \in \mathbb{Q}$ for $i \in\{1,2, \ldots, n\}$. It gives a contradiction to Lemma 5.3 . 


\section{$6 \quad$ Further questions}

In Theorem 3.1, we assumed that the expansion factor $\lambda$ is irrational and Pisot. If $\lambda$ is rational, which means it is a natural number, there may be arbitrarily long arithmetic progressions even if we fix the distance $x \in \mathbb{R}^{d} \backslash\{0\}$, as we have seen in Example 2.7 and Example 2.8. However, we do not understand the situation where we drop the Pisot assumption. We do not know any examples with irrational non-Pisot expansion factors that admit one-dimensional arithmetic progressions of arbitrary length. Moreover, the existence and absence of infinite one-dimensional arithmetic progressions may depend on the choice of a tiling from the hull. For example, we do not know if there is a tiling in the hull of the table substitution that does not admit infinite one-dimensional arithmetic progressions.

The study of full-rank infinite arithmetic progressions in Section 4 is also not complete. We understand the self-similar case, but for general $Q$, there are still open problems. For example, we suspect if all the eigenvalues for $Q$ are integers and the tiling has pure discrete dynamical spectrum, then the tiling is limit-periodic, but we do not have a proof for this. We have assumed several conditions, such as that $Q(L) \subset L$ in Theorem 4.7. We do not know what happens if we drop these assumptions.

\section{Appendix}

Here, we put some proofs in the main part of the paper.

\subsection{The proof for Proposition 4.13}

First we prove Proposition 4.13

Proof. We set $M=e \max \left\{n_{1}, n_{2}, \ldots, n_{e}\right\}$ and prove that this $M$ satisfies the desired condition. To prove this, take arbitrary $k_{1}, k_{2}, \ldots, k_{e} \in \mathbb{Z}$ and we shall prove that $\sum_{i=1}^{e} k_{i} v_{i}$ is included in $\Xi+\Xi+\cdots+\Xi$ (the result of the summation for $M \Xi$ 's). We use a tile map $f: \mathcal{T} \rightarrow \mathcal{T}$ which was used to define a control points map $c$. We can assume that $T$ and $f(T)$ are always the same tile type. For any $i=1,2, \ldots, e$ such that $k_{i} \geqq 0$, by considering $n_{i}$-adic expansion of $k_{i}$, there are $k_{i, 0}, k_{i, 1}, \ldots, k_{i, N_{i}} \in\left\{0,1, \ldots n_{i}-1\right\}$ such that

$$
k_{i}=\sum_{j=0}^{N_{i}} k_{i, j} n_{i}^{N_{i}-j} .
$$

For $i$ with $k_{i}<0$, similarly there are $k_{i, 0}, k_{i, 1}, \ldots, k_{i, N_{i}} \in\left\{0,1, \ldots, n_{i}\right\}$ such that

$$
-k_{i}=\sum_{j=0}^{N_{i}} k_{i, j} n_{i}^{N_{i}-j} .
$$


Since each $N_{i}$ can be replaced with a larger integer, we may assume that $N_{1}=N_{2}=\cdots N_{e}$. Let us denote this number by $N$. Set $I_{+}=\left\{i=1,2, \ldots, e \mid k_{i} \geqq 0\right\}$ and $I_{-}=\{i=$ $\left.1,2, \ldots, e \mid k_{i}<0\right\}$.

For each $i \in I_{+}$and $l=1,2, \ldots, n_{i}-1$, we shall choose tiles

$$
S_{N+1, l, i}, S_{0, l, i}^{\prime}
$$

and for each $i \in I_{-}$and $l=1,2, \ldots, n_{i}$, we shall chose tiles

$$
T_{N+1, l, i}, T_{0, l, i}^{\prime},
$$

all in $\mathcal{T}$ and with the same tile type, such that

$$
\sum_{i \in I_{+}} \sum_{l=1}^{n_{i}-1}\left(c\left(S_{N+1, l, i}\right)-c\left(S_{0, l, i}^{\prime}\right)\right)-\sum_{i \in I_{-}} \sum_{l=1}^{n_{i}-1}\left(c\left(T_{N+1, l, i}\right)-c\left(T_{0, l, i}^{\prime}\right)\right)=\sum_{i=1}^{e} k_{i} v_{i} .
$$

Since all objects $c\left(S_{N+1, l, i}\right)-c\left(S_{0, l, i}^{\prime}\right)$ and $c\left(T_{N+1, l, i}\right)-c\left(T_{0, l, i}^{\prime}\right)$, where $i=1,2, \ldots, e$ and $l=1,2, \ldots n_{i}-1$, are members of $\Xi$, and the number of these objects is at most $M$, once we have proved this equation (15), we finish the proof of this proposition.

First, take $i \in I_{+}$and $l=1,2, \ldots n_{i}-1$, and we shall construct $S_{N+1, l, i}$ and $S_{0, l, i}^{\prime}$. The latter is defined by $S_{0, l, i}^{\prime}=f^{N+1}\left(T^{(0)}\right)$. To construct the former, set $S_{0, l, i}=T^{(0)}$ and we choose $S_{1, l, i}, S_{2, l, i}, \ldots, S_{N+1, l, i}$ inductively, as follows. Given an $S_{j, l, i}$ of the same tile type as $T^{(0)}$, by assumption we can take an $S_{j+1, l, i} \in \omega\left(S_{j, l, i}\right)$ of the same type as $T^{(0)}$ such that

$$
c\left(S_{j+1, l, i}\right)-Q\left(c\left(S_{j, l, i}\right)\right)= \begin{cases}v_{i} & \text { if } k_{i, j} \geqq l \\ 0 & \text { otherwise. }\end{cases}
$$

By induction, we have found $S_{N+1, l, i}$.

Similarly, for each $i \in I_{-}$and $l=1,2, \ldots n_{i}-1$, we can take $T_{0, l, i}=T^{(0)}, T_{1, l, i}, \ldots, T_{N+1, l, i} \in$ $\mathcal{T}$ of the same type so that

$$
c\left(T_{j+1, l, i}\right)-Q\left(c\left(T_{j, l, i}\right)\right)= \begin{cases}v_{i} & \text { if } k_{i, j} \geqq l \\ 0 & \text { otherwise }\end{cases}
$$

By induction, we have defined $T_{N+1, l, i} . T_{0, l, i}^{\prime}$ is defined as $f^{N+1}\left(T_{0, l, i}\right)$.

Now we have defined the necessary tiles, and we shall prove the equation (15). By using the fact that

$$
\left.\sum_{l=1}^{n_{i}-1}\left(c\left(S_{j, l, i}\right)-Q\left(c\left(S_{j-1, l, i}\right)\right)\right)\right)=k_{i, j-1} v_{i}
$$


we have the following:

$$
\begin{aligned}
\sum_{l=1}^{n_{i}-1}\left(c\left(S_{N+1, l, i}\right)-c\left(S_{0, l, i}^{\prime}\right)\right) & =\sum_{l=1}^{n_{i}-1}\left(c\left(S_{N+1, l, i}\right)-Q^{N+1}\left(c\left(S_{0, l, i}\right)\right)\right) \\
& =\sum_{j=1}^{N+1} \sum_{l=1}^{n_{i}-1} Q^{N-j+1}\left(c\left(S_{j, l, i}\right)-Q\left(c\left(S_{j-1, l, i}\right)\right)\right) \\
& =\sum_{j=1}^{N+1} Q^{N-j+1}\left(k_{i, j-1} v_{i}\right) \\
& =\sum_{j=1}^{N+1} n_{i}^{N-j+1} k_{i, j-1} v_{i} \\
& =k_{i} v_{i} .
\end{aligned}
$$

Also, we have an equation

$$
\sum_{l=1}^{n_{i}-1}\left(c\left(T_{N+1, l, i}\right)-c\left(T_{0, l, i}^{\prime}\right)\right)=-k_{i} v_{i} .
$$

These two equations prove the equation (15).

\subsection{The proof for Theorem 4.18}

Here, we prove Theorem 4.18 ,

Let $\omega$ be a primitive FLC substitution rule with an expansion map $Q$ and an alphabet $\mathcal{A}$. For each $P \in \mathcal{A}$, the patch $\omega(P)$ is the result of "expanding $P$ by $Q$ and then subdividing it". Henceforth we also use a "subdividing without expanding" map $\sigma$. By using this $\sigma$ instead of $\omega$, the proof is simplified. The map $\sigma$ is defined via

$$
\sigma(P)=Q^{-1}(\omega(P))
$$

(See Figure 5,) Here, we set $Q^{k}(T)=\left(Q^{k}(S), l\right)$ for each labeled tile $T=(S, l)$ and $k \in \mathbb{Z}$, and $Q^{k}(\mathcal{P})=\left\{Q^{k}(T) \mid T \in \mathcal{P}\right\}$ for each patch $\mathcal{P}$. (For unlabeled tiles $T, Q^{k}(T)$ has the usual meaning.) We will also "subdivide without expanding" $Q^{l}(P)+x$ 's, where $l \in \mathbb{Z}$, $x \in \mathbb{R}^{d}$ and $P \in \mathcal{A}$, and so define

$$
\sigma\left(Q^{l}(P)+x\right)=Q^{l-1}(\omega(P))+x .
$$

For each patch $\mathcal{P}$ of which elements are of the form $Q^{l}(P)+x$, we set

$$
\sigma(\mathcal{P})=\bigcup_{T \in \mathcal{P}} \sigma(T)
$$

Then we have the following properties: 


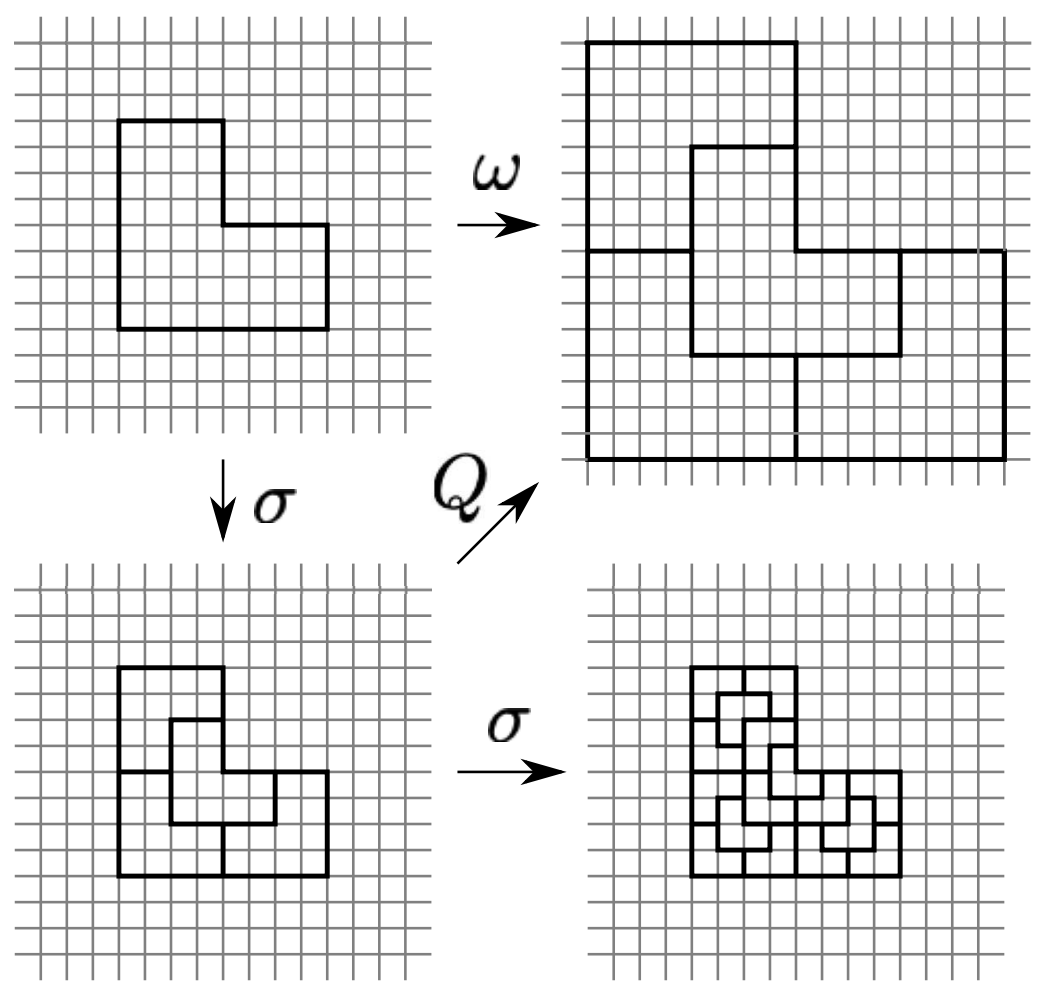

Figure 5: The chair substitution $\omega$ and the corresponding subdivision map $\sigma$ 
1. $\sigma(\mathcal{P})$ is also a patch consisting of tiles of the form $Q^{l}(P)+x$ and $\operatorname{supp} \sigma(\mathcal{P})=\operatorname{supp} \mathcal{P}$.

2. $\sigma \circ Q^{l}=Q^{l} \circ \sigma$ for any $l \in \mathbb{Z}$.

We now assume Setting 2 in Subsection 4.2 and the self-affine tiling $\mathcal{T}$ has pure discrete dynamical spectrum and satisfies the equivalent conditions in Corollary 4.10, To prove Theorem 4.18, we divide the proof into lemmas. First, by using Lemma 4.3 and replacing $\omega$ with its power, without loss of generality we may assume that, for each overlap $(S, x, T)$ for $\mathcal{T}$, we have

$$
\omega(S+x) \cap \omega(T) \neq \emptyset,
$$

which is equivalent to

$$
\sigma(S+x) \cap \sigma(T) \neq \emptyset .
$$

Set $V_{\max }=\max _{P \in \mathcal{A}} \operatorname{vol}(P), V_{\min }=\min _{P \in \mathcal{A}} \operatorname{vol}(P)$ and $D=|\operatorname{det} Q|$. (Here, vol denotes the Lebesgue measure.)

In what follows we tacitly use the following result on the boundaries $\partial T$ of the tiles $T$ in self-affine tilings:

Lemma 7.1 ([23]). Let $T$ be a tile in a self-affine tiling. Then $\operatorname{vol}(\partial T)=0$, where vol denotes the Lebesgue measure and $\partial$ denotes the boundary.

Lemma 7.2. For an overlap $\left(S_{0}, y_{0}, T_{0}\right)$ and $k \in \mathbb{Z}_{>0}$, set

$$
K_{k}=\operatorname{supp}\left(\sigma^{k}\left(S_{0}+y_{0}\right) \cap \sigma^{k}\left(T_{0}\right)\right) .
$$

Then, for each $k, K_{k} \subset K_{k+1}$ and

$$
1-\frac{\operatorname{vol}\left(K_{k+1}\right)}{\operatorname{vol}\left(\left(\operatorname{supp} S_{0}+y_{0}\right) \cap \operatorname{supp} T_{0}\right)} \leqq\left(1-\frac{V_{\text {min }}}{D V_{\max }}\right)\left(1-\frac{\operatorname{vol}\left(K_{k}\right)}{\operatorname{vol}\left(\left(\operatorname{supp} S_{0}+y_{0}\right) \cap \operatorname{supp} T_{0}\right)}\right),
$$

and so $\operatorname{vol}\left(K_{k}\right) \rightarrow \operatorname{vol}\left(\left(\operatorname{supp} S_{0}+y_{0}\right) \cap \operatorname{supp} T_{0}\right)$ as $k \rightarrow \infty$.

Proof. If $k>0, S \in \sigma^{k}\left(S_{0}+y_{0}\right), T \in \sigma^{k}\left(T_{0}\right)$, int $S \cap \operatorname{int} T \neq \emptyset$ and $S \neq T$, then there is a $U_{S, T} \in \sigma(S) \cap \sigma(T)$. It is clear that $\operatorname{int} U_{S, T} \cap K_{k}=\emptyset$, since otherwise $S=T$. We have

$$
\begin{array}{r}
1-\frac{\operatorname{vol}\left(K_{k+1}\right)}{\operatorname{vol}\left(\left(\operatorname{supp} S_{0}+y_{0}\right) \cap \operatorname{supp} T_{0}\right)} \leqq 1-\frac{\operatorname{vol}\left(K_{k}\right)}{\operatorname{vol}\left(\left(\operatorname{supp} S_{0}+y_{0}\right) \cap \operatorname{supp} T_{0}\right)} \\
-\frac{1}{\operatorname{vol}\left(\left(\operatorname{supp} S_{0}+y_{0}\right) \cap \operatorname{supp} T_{0}\right)} \sum \operatorname{vol}\left(\operatorname{supp} U_{S, T}\right),
\end{array}
$$


where the sum is for all $S$ and $T$ with the above condition. The claim of the lemma follows from the observations

$$
\begin{array}{r}
D^{-k} V_{\max } \operatorname{card}\left\{(S, T) \in \sigma^{k}\left(S_{0}+y_{0}\right) \cap \sigma^{k}\left(T_{0}\right) \mid T \neq S, \operatorname{int} T \cap \operatorname{int} S \neq \emptyset\right\} \\
\geqq \operatorname{vol}\left(\left(\operatorname{supp}\left(S_{0}+y_{0}\right) \cap \operatorname{supp} T_{0}\right) \backslash K_{k}\right),
\end{array}
$$

and

$$
\operatorname{vol}\left(\operatorname{supp} U_{S, T}\right) \geqq D^{-k-1} V_{\min }
$$

Set

$$
\mathcal{P}_{k}=\bigcap_{x \in \Xi} \sigma^{k}(\mathcal{T}-x)
$$

Note that $Q^{k}\left(\mathcal{P}_{k}\right)=\bigcap_{x \in \Xi} D_{Q^{k}(x)}$. We will show that (1)any $T \in Q^{k}\left(\mathcal{P}_{k}\right)$ is a part of a full-rank infinite arithmetic progression, and (2)the density of $\mathcal{P}_{k}$, which is same as the density of $Q^{k}\left(\mathcal{P}_{k}\right)$, tends to 1 as $k \rightarrow \infty$.

Note that by Lemma 7.2, we have $\operatorname{supp} \mathcal{P}_{k} \subset \operatorname{supp} \mathcal{P}_{k+1}$ for each $k>0$.

Lemma 7.3. There is a natural number $M$ such that for any $k>0$ and $S \in \mathcal{P}_{k}$, the tile $Q^{k} S$ is a tile in $\mathcal{T}$ with $\left\{Q^{k} S+x \mid x \in Q^{k+M} L\right\} \subset \mathcal{T}$.

Proof. By assumption, there is an $M$ such that

$$
Q^{M} L \subset \Xi
$$

If $k>0$ and $S \in \mathcal{P}_{k}$, then for each $x \in \Xi$ we have $S+x \in \sigma^{k}(\mathcal{T})$. By the definition of $\sigma$, we see $Q^{k}(S)+Q^{k}(x) \in Q^{k} \sigma^{k}(\mathcal{T})=\omega^{k}(\mathcal{T})=\mathcal{T}$. By (19), we can set $x=Q^{M}(y)$ for each $y \in L$.

Lemma 7.4. If $T \in \mathcal{T}$ does not satisfy $\left\{T+x \mid x \in Q^{l}(L)\right\} \subset \mathcal{T}$ for any $l>0$, then $T \notin Q^{k} \mathcal{P}_{k}$ for any $k$.

Proof. This is clear by Lemma 7.3 ,

By this lemma, the proof of Theorem 4.18 is completed if we prove the density of each $\mathcal{P}_{k}$ is large enough. We now show this.

Lemma 7.5. For all van Hove sequence $\left(A_{n}\right)_{n}$, we have

$$
\lim _{k \rightarrow \infty} \operatorname{dens}_{\left(A_{n}\right)_{n}} \mathcal{T} \backslash Q^{k} \mathcal{P}_{k}=0 .
$$

(In fact, the convergence as $k \rightarrow \infty$ is uniform for all van Hove sequence $\left(A_{n}\right)_{n}$.) 
Proof. For each $S \in \mathcal{T}$ and $x \in \Xi$, by Lemma 7.2 we have, as $k \rightarrow \infty$,

$$
\begin{aligned}
\operatorname{vol} \operatorname{supp}\left(\sigma^{k}(S) \cap \sigma^{k}(\mathcal{T}-x)\right) & =\operatorname{vol} \operatorname{supp}\left(\bigcup_{T \in \mathcal{T} \sqcap(\operatorname{int} S+x)} \sigma^{k}(S) \cap \sigma^{k}(T-x)\right) \\
& =\sum_{T \in \mathcal{T} \sqcap(\operatorname{int} S+x)} \operatorname{vol} \operatorname{supp}\left(\sigma^{k}(S) \cap \sigma^{k}(T-x)\right) \\
& \rightarrow \sum_{T \in \mathcal{T} \sqcap(\operatorname{int} S+x)} \operatorname{vol}(\operatorname{supp} S \cap(\operatorname{supp} T-x)) \\
& =\operatorname{vol} \operatorname{supp} S .
\end{aligned}
$$

Since there are only finitely many equivalence classes of overlaps, this convergence is uniform in $S$ and $x$.

We use the fact that the overlaps are finite up to equivalence, for one more time. Take an $S \in \mathcal{T}$ and fix it. Consider a map

$$
\Xi \ni x \mapsto\{[S, x, T] \mid T \in \mathcal{T} \sqcap(\text { int } S+x)\} .
$$

Since the range of this map is a finite set, there is a finite set $F_{S} \subset \Xi$ such that for any $x \in \Xi$ there is a $y \in F_{S}$ with

$$
\{[S, x, T] \mid T \in \mathcal{T} \sqcap(\operatorname{int} S+x)\}=\{[S, y, T] \mid T \in \mathcal{T} \sqcap(\operatorname{int} S+y)\} .
$$

Note that card $F_{S} \leqq N$ for some constant $N$ independent of $S$, where $N=2^{N_{0}}$ and $N_{0}$ is the number of all equivalence classes of overlaps in $\mathcal{T}$. ( $N$ is the number of all subsets of the set of the equivalence classes of overlaps.) We will use this estimate for the estimate of the density of $\sigma^{k}(\mathcal{T}) \backslash \mathcal{P}_{k}$, as follows.

Let $\varepsilon$ be an arbitrary positive real number. There is a natural number $k_{0}$ such that if $k \geqq k_{0}, S \in \mathcal{T}$ and $x \in \Xi$, we have

$$
0 \leqq \operatorname{vol} \operatorname{supp} S-\operatorname{vol} \operatorname{supp}\left(\sigma^{k}(S) \cap \sigma^{k}(\mathcal{T}-x)\right)=\operatorname{vol} \operatorname{supp}\left(\sigma^{k}(S) \backslash \sigma^{k}(\mathcal{T}-x)\right)<\varepsilon .
$$

For any van Hove sequence $\left(A_{n}\right)_{n}$ and $k \geqq k_{0}$,

$$
\begin{aligned}
& \limsup _{n \rightarrow \infty} \frac{1}{\operatorname{vol}\left(A_{n}\right)} \operatorname{vol} \operatorname{supp}\left(\left(\sigma^{k}(\mathcal{T}) \backslash \mathcal{P}_{k}\right) \wedge A_{n}\right) \\
& =\limsup _{n} \frac{1}{\operatorname{vol}\left(A_{n}\right)} \sum_{T \in \mathcal{T} \wedge A_{n}} \operatorname{vol} \operatorname{supp}\left(\sigma^{k}(T) \backslash \mathcal{P}_{k}\right) \\
& \leqq \limsup _{n} \frac{1}{\operatorname{vol}\left(A_{n}\right)} \sum_{T \in \mathcal{T} \wedge A_{n}} \sum_{x \in F_{T}} \operatorname{vol} \operatorname{supp}\left(\sigma^{k}(T) \backslash \sigma^{k}(\mathcal{T}-x)\right) \\
& \leqq \limsup _{n} \frac{1}{\operatorname{vol}\left(A_{n}\right)} \sum_{T \in \mathcal{T} \wedge A_{n}} N \varepsilon \\
& \leqq \frac{N \varepsilon}{V_{\min }}
\end{aligned}
$$


where we used the fact that

$$
\begin{aligned}
\sigma^{k}(T) \backslash \mathcal{P}_{k} & =\bigcup_{x \in \Xi} \sigma^{k}(T) \backslash \sigma^{k}(\mathcal{T}-x) \\
& =\bigcup_{x \in F_{T}} \sigma^{k}(T) \backslash \sigma^{k}(\mathcal{T}-x)
\end{aligned}
$$

and

$$
V_{\text {min }} \operatorname{card} \mathcal{T} \wedge A_{n} \leqq \operatorname{vol}\left(A_{n}\right)
$$

We now finish the proof. Take a van Hove sequence $\left(A_{n}\right)_{n}$. For each $k \geqq k_{0},\left(Q^{-k} A_{n}\right)_{n}$ is again a van Hove sequence. By $\mathcal{T}=\omega^{k}(\mathcal{T})=Q^{k} \sigma^{k}(\mathcal{T})$, we have

$$
\begin{aligned}
\operatorname{dens}_{\left(A_{n}\right)_{n}} \mathcal{T} \backslash Q^{k} \mathcal{P}_{k} & =\limsup _{n} \frac{1}{\operatorname{vol}\left(A_{n}\right)} \operatorname{vol} \operatorname{supp}\left(\left(\mathcal{T} \backslash Q^{k} \mathcal{P}_{k}\right) \wedge A_{n}\right) \\
& =\limsup _{n} \frac{1}{\operatorname{vol}\left(Q^{-k} A_{n}\right)} \operatorname{vol} \operatorname{supp}\left(\left(\sigma^{k}(\mathcal{T}) \backslash \mathcal{P}_{k}\right) \wedge Q^{-k} A_{n}\right) \\
& \leqq \frac{N \varepsilon}{V_{\text {min }}},
\end{aligned}
$$

and so $\operatorname{dens}_{\left(A_{n}\right)_{n}} \mathcal{T} \backslash Q^{k} \mathcal{P}_{k}$ tends to 0 as $k \rightarrow \infty$.

\section{Acknowledgment}

YN was supported by EPSRC grant EP/S010335/1. SA was supported by JSPS grants (17K05159, 17H02849, BBD30028). JL was supported by NRF grant No. 2019R1I1A3A01060365. This study was led by YN. The authors thank the referee for valuable comments. The authors also thank Dan Rust for the check of English in section 1 and 2.

\section{References}

[1] S. Akiyama and J.-Y. Lee, Algorithm for determining pure pointedness of self-affine tilings, Advances in Mathematics 226.4 (2011), 2855-2883.

[2] S. Akiyama and J.-Y. Lee, Overlap coincidence to strong coincidence in substitution tiling dynamics, European J. Combin. 39 (2014), 233-243.

[3] M. Baake and U. Grimm, Aperiodic order Vol. 1, Cambridge University Press (2013).

[4] M. Baake and and U. Grimm, Diffraction of limit periodic point sets, Philosophical Magazine, 91(19-21) (2011), 2661-2670. 
[5] M. Baake, R. V. Moody and M. Schlottmann, Limit-(quasi) periodic point sets as quasicrystals with p-adic internal spaces, Journal of Physics A: Mathematical and General 31.27 (1998), 5755.

[6] M. Baake and D. Lenz, Dynamical systems on translation bounded measures: Pure point dynamical and diffraction spectra, Ergodic Theory and Dynam. Sys., 24.6 (2004), 1867-1893.

[7] D. Frettlöh, Nichtperiodische Pflasterungen mit ganzzahligem Inflationsfaktor, Ph.D. thesis, Universität Dortmund (2002).

[8] H. Furstenberg, Recurrence in ergodic theory and combinatorial number theory, Princeton University Press (2014).

[9] F. Gähler and R. Klitzing, The diffraction pattern of self-similar tilings, NATO ASI Series C Mathematical and Physical Sciences-Advanced Study Institute, 489 (1997),141174 .

[10] C. Godreche, The sphinx: a limit-periodic tiling of the plane, Journal of Physics A: Mathematical and General 22.24 (1989): L1163.

[11] J-B. Gouéré, Quasicrystals and almost periodicity, Communications in mathematical physics 255.3 (2005), 655-681.

[12] A. Iwanik, and Y. Lacroix, Some constructions of strictly ergodic non-regular Toeplitz flows, Studia Math 110.2 (1994): 191-203.

[13] K. Jacobs and M. Keane, 0-1-sequences of Toeplitz type, Zeitschrift für Wahrscheinlichkeitstheorie und Verwandte Gebiete 13.2 (1969), 123-131.

[14] A. Klick, N. Strungaru and A. Tcaciuc, On arithmetic progressions in model sets, arXiv:2003.13860

[15] J.-Y. Lee, Substitution Delone sets with pure point spectrum are inter-model sets, Journal of Geometry and Physics, 57(11) (2007), 2263-2285.

[16] J.-Y. Lee and B. Solomyak, Pure point diffractive substitution Delone sets have the Meyer property, Discr. Comput. Geom. 39 (2008), 319-338.

[17] D. Lenz, T. Spindeler and N. Strungaru, Pure point diffraction and mean, Besicovitch and Weyl almost periodicity, arXiv:2006.10821

[18] J.-Y. Lee and B. Solomyak, Pisot family substitution tilings, discrete spectrum and the Meyer property, Discr. Conti. Dynam. Sys., 32-3 (2012), 935-959. 
[19] J.-Y. Lee, R. V. Moody, and B. Solomyak, Consequences of pure point diffraction spectra for multiset substitution systems, Discr. Comput. Geom. 29.4 (2003), 525560 .

[20] R. de la Llave and A. Windsor, An application of topological multiple recurrence to tiling, Discr. Conti. Dynam. Sys. -S, 2(2) (2009), 315-324.

[21] Neil Mañibo, Lyapunov exponents in the spectral theory of primitive inflation systems, Ph. D. Thesis, Universität Bielefeld, 2019.

[22] Y. Nagai, The common structure for objects in aperiodic order and the theory of local matching topology, arXiv:1811.04642

[23] B. Praggastis, Markov partitions for hyperbolic toral automorphisms, Ph.D. Thesis, University of Washington, (1992).

[24] B. Praggastis, Numeration systems and Markov partitions from self similar tilings, Trans. Amer. Math. Soc. 351 (1999), no. 8, 3315-3349.

[25] M. Queffélec, Substitution dynamical systems-spectral analysis, Vol. 1294. Springer, 2010 .

[26] B. Solomyak, Eigenfunctions for substitution tiling systems, Probability and Number Theory, Kanazawa 2005 (pp. 433-454), Mathematical Society of Japan (2007).

[27] B. Solomyak, Dynamics of self-similar tilings, Ergodic Theory and Dynam. Sys., 17-3 (1997), 695-738. 